\title{
Transfer principle in quantum set theory
}

\author{
Masanao OzaWA \\ Graduate School of Information Sciences \\ Tôhoku University, Aoba-ku, Sendai, 980-8579, Japan
}

\begin{abstract}
In 1981, Takeuti introduced quantum set theory as the quantum counterpart of Boolean valued models of set theory by constructing a model of set theory based on quantum logic represented by the lattice of closed subspaces in a Hilbert space and showed that appropriate quantum counterparts of ZFC axioms hold in the model. Here, Takeuti's formulation is extended to construct a model of set theory based on the logic represented by the lattice of projections in an arbitrary von Neumann algebra. A transfer principle is established that enables us to transfer theorems of ZFC to their quantum counterparts holding in the model. The set of real numbers in the model is shown to be in one-to-one correspondence with the set of self-adjoint operators affiliated with the von Neumann algebra generated by the logic. Despite the difficulty pointed out by Takeuti that equality axioms do not generally hold in quantum set theory, it is shown that equality axioms hold for any real numbers in the model. It is also shown that any observational proposition in quantum mechanics can be represented by a corresponding statement for real numbers in the model with the truth value consistent with the standard formulation of quantum mechanics, and that the equality relation between two real numbers in the model is equivalent with the notion of perfect correlation between corresponding observables (self-adjoint operators) in quantum mechanics. The paper is concluded with some remarks on the relevance to quantum set theory of the choice of the implication connective in quantum logic.
\end{abstract}

\section{Introduction}

Since Birkhoff and von Neumann [2] introduced quantum logic in 1936 as the semantical structure of propositional calculus for observational propositions on a quantum system, there have been continued research efforts for introducing the methods of symbolic logic into the logical structure of our recognition on quantum systems. However, the introduction of basic notions of sets and numbers in quantum logic was not realized before Takeuti 35] introduced quantum set theory in his seminal paper published in 1981.

Quantum set theory has two main origins, quantum logic introduced by Birkhoff and von Neumann [2] and Boolean valued models of set theory by which Scott and Solovay [29] reformulated the method of forcing invented by Cohen [4, 5] for the independence proof of the continuum hypothesis. Birkhoff and von Neumann 2 argued that the propositional

calculus for quantum mechanics is represented by the lattice of closed linear subspaces of the Hilbert space of state vectors of the system so that logical operations correspond to lattice operations of the subspaces such as set intersection (conjunction), closure of space 
sum (disjunction), and orthogonal complement (negation), whereas the propositional calculus for classical mechanics is represented by a Boolean logic corresponding to the Boolean algebra of Borel subsets of the phase space of the physical system modulo the sets of Lebesgue measure zero so that logical operations correspond to set operations in an obvious way.

The correspondence between classical mechanics and classical logic can be extended nowadays to its ultimate form using Boolean valued analysis introduced by Scott 28 and developed by Takeuti [32, 33, 34, 37, 36, 38, and many followers [6, 11, 14, 15, 16, 17, 18, 19, 20, 21, 22, 23, 24, 25, 30] as follows. Let $V^{(\mathcal{B})}$ be the Boolean valued universe of set theory constructed from the complete Boolean algebra $\mathcal{B}$ of Borel subsets of the phase space of a classical mechanical system modulo the sets with Lebesgue measure zero. Then, there is a natural correspondence between physical quantities of the system and the real numbers in the model $V^{(\mathcal{B})}$, and by the ZFC transfer principle for Boolean valued models, any theorem on real numbers provable in $\mathrm{ZFC}$ gives rise to a valid physical statement on physical quantities of that system.

One of the interesting programs in constructing quantum set theory is to extend this correspondence to that between quantum mechanics and quantum set theory. Let $V^{(\mathcal{Q})}$ be the universe of set theory constructed from the complete orthomodular lattice $\mathcal{Q}$ of projections in a von Neumann algebra $\mathcal{M}$ of observables of a physical system [3]. Then, it is expected that there is a natural correspondence between physical quantities (observables) of the system and the real numbers in the model $V^{(\mathcal{Q})}$, and it is also expected that we have a suitable transfer principle from theorems in ZFC to a valid physical statement on physical quantities of that system.

When the von Neumann algebra $\mathcal{M}$ is abelian, the corresponding physical system is considered as a classical system, and the projection lattice $\mathcal{Q}$ is a complete Boolean algebra, so that $V^{(\mathcal{Q})}$ is nothing but a Boolean valued model of set theory.

In Ref. 35, Takeuti investigated in the case where the von Neumann algebra is a type I factor or equivalently the algebra of all bounded operators on a Hilbert space $\mathcal{H}$. He showed that axioms of ZFC can be transferred to appropriate valid statements in $V^{(\mathcal{Q})}$ and that real numbers in $V^{(\mathcal{Q})}$ naturally correspond to self-adjoint operators on $\mathcal{H}$ or equivalently observables of the quantum system described by $\mathcal{H}$.

In this paper, we extend Takeuti's investigation to an arbitrary von Neumann algebra $\mathcal{M}$ on a Hilbert space $\mathcal{H}$. Instead of transferring each axiom of ZFC to an appropriate valid statement in $V^{(\mathcal{Q})}$, this paper establishes a unified transfer principle from theorems of ZFC to valid statements in $V^{(\mathcal{Q})}$, which enables us to directly obtain transferred theorems in $V^{(\mathcal{Q})}$ without proving them from the transferred axioms in $V^{(\mathcal{Q})}$. The only restriction to our transfer principle is that a theorem in ZFC to transfer should be expressed by a $\Delta_{0}$-formula in the language of set theory; if $\mathcal{M}$ is abelian, it is known from a fundamental theorem of Boolean valued models of set theory that this restriction can be removed.

After establishing the transfer principle, we investigate real numbers in $V^{(\mathcal{Q})}$. It is rather easy to extend Takeuti's result to show that there is a one-to-one correspondence between real numbers in $V^{(\mathcal{Q})}$ and self-adjoint operators on $\mathcal{H}$ affiliated with $\mathcal{M}$ or naturally observables of the physical system described by $\mathcal{M}$. Another important result about the real numbers in $V^{(\mathcal{Q})}$ concerns the equality between real numbers. As previously shown by Takeuti [35], one of the difficult aspects of quantum set theory is that 
equality axioms do not generally hold. Since the equality is one of the most fundamental relations, the lack of equality axioms has led many researchers to question the applicability of quantum set theory to a real science such as quantum mechanics. Here, we shall show that the equality axioms generally hold between any real numbers in $V^{(\mathcal{Q})}$. We also show that the equality between two real numbers is equivalent to the notion of quantum perfect correlation [26, 27] between two corresponding observables in the quantum system. Thus, despite the above difficulty of the equality in $V^{(\mathcal{Q})}$, the equality between real numbers in $V^{(\mathcal{Q})}$ has a clear physical meaning, and thus quantum set theory is expected to play a crucial role in the future investigations in the interpretation of quantum mechanics.

Section 2 collects results on quantum logic used in the later sections. Section 3 introduces the model $V^{(\mathcal{Q})}$ of (quantum) set theory based on the projection lattice $\mathcal{Q}$ of a von Neumann algebra $\mathcal{M}$, and proves some basic properties. Section 4 is devoted to proving the ZFC Transfer Principle that states that any theorem of ZFC expressed by a $\Delta_{0}$-formula holds in the model $V^{(\mathcal{Q})}$ up to the well-defined truth value in $\mathcal{Q}$ representing the degree of commutativity of elements in $V^{(\mathcal{Q})}$ appearing in that formula. Section 5 introduces real numbers in the model $V^{(\mathcal{Q})}$, and proves that the equality axioms hold for the reals in $V^{(\mathcal{Q})}$. Section 6 proves that the set $\mathbf{R}^{(\mathcal{Q})}$ of reals in $V^{(\mathcal{Q})}$ is in one-to-one correspondence with the set of self-adjiont operators affiliated with the von Neumann algebra $\mathcal{M}$ generated by the logic $\mathcal{Q}$, and shows that through this correspondence observationally valid propositions on the physical system is naturally expressed as the valid statements on the reals in $V^{(\mathcal{Q})}$. This section also proves that the equality relation between two reals in $V^{(\mathcal{Q})}$ is equivalent with the notion of the quantum perfect correlation between the corresponding observables (self-adjoint operators), a notion recently introduced into quantum mechanics [26, 27]. Section 7 concludes the present paper with discussions on an open problem on the choice of the implication connective.

\section{Quantum logic}

Let $\mathcal{H}$ be a Hilbert space. For any subset $S \subseteq \mathcal{H}$, we denote by $S^{\perp}$ the orthogonal complement of $S$, i.e., $S^{\perp}=\{\psi \in \mathcal{H} \mid\langle\xi, \psi\rangle=0$ for all $\xi \in S\}$. Then, $S^{\perp \perp}$ is the closed linear span of $S$. Let $\mathcal{C}(\mathcal{H})$ be the set of all closed linear subspaces in $\mathcal{H}$. With the set inclusion $M \subseteq N$ as the partial ordering, the set $\mathcal{C}(\mathcal{H})$ is a complete lattice. The lattice operations on $\mathcal{C}(\mathcal{H})$ are characterized by $M \wedge N=M \cap N, M \vee N=(M \cup N)^{\perp \perp}$, $\wedge \mathcal{S}=\bigcap \mathcal{S}$, and $\vee \mathcal{S}=(\bigcup \mathcal{S})^{\perp \perp}$ for any $\mathcal{S} \subseteq \mathcal{C}(\mathcal{H})$. The operation $M \mapsto M^{\perp}$ is an orthocomplementation on the lattice $\mathcal{C}(\mathcal{H})$, with which $\mathcal{C}(\mathcal{H})$ is a complete orthomodular lattice [12, p. 65].

Denote by $\mathcal{B}(\mathcal{H})$ the algebra of bounded linear operators on $\mathcal{H}$ and $\mathcal{Q}(\mathcal{H})$ the set of projections on $\mathcal{H}$. We define the operator ordering on $\mathcal{B}(\mathcal{H})$ by $A \leq B$ iff $\langle\psi, A \psi\rangle \leq$ $\langle\psi, B \psi\rangle$ for all $\psi \in \mathcal{H}$. For any $A \in \mathcal{B}(\mathcal{H})$, denote by $\mathcal{R}(A) \in \mathcal{C}(\mathcal{H})$ the closure of the range of $A$, i.e., $\mathcal{R}(A)=(A \mathcal{H})^{\perp \perp}$. For any $M \in \mathcal{C}(\mathcal{H})$, denote by $\mathcal{P}(M) \in \mathcal{Q}(\mathcal{H})$ the projection operator of $\mathcal{H}$ onto $M$. Then, $\mathcal{R} \mathcal{P}(M)=M$ for all $M \in \mathcal{C}(\mathcal{H})$ and $\mathcal{P} \mathcal{R}(P)=P$ for all $P \in \mathcal{Q}(\mathcal{H})$, and we have $P \leq Q$ if and only if $\mathcal{R}(P) \subseteq \mathcal{R}(Q)$ for all $P, Q \in \mathcal{Q}(\mathcal{H})$, so that $\mathcal{Q}(\mathcal{H})$ with the operator ordering is also a complete orthomodular lattice isomorphic to $\mathcal{C}(\mathcal{H})$. The lattice operations are characterized by $P \wedge Q=\lim _{n \rightarrow \infty}(P Q)^{n}, P^{\perp}=1-P$ 
for all $P, Q \in \mathcal{Q}(\mathcal{H})$.

Let $\mathcal{A} \subseteq \mathcal{B}(\mathcal{H})$. We denote by $\mathcal{A}^{\prime}$ the commutant of $\mathcal{A}$ in $\mathcal{B}(\mathcal{H})$. A self-adjoint subalgebra $\mathcal{M}$ of $\mathcal{B}(\mathcal{H})$ is called a von Neumann algebra on $\mathcal{H}$ iff $1 \in \mathcal{M}$ and $\mathcal{M}^{\prime \prime}=\mathcal{M}$. By the double commutation theorem [31, a self-adjoint algebra $\mathcal{M} \subseteq \mathcal{B}(\mathcal{H})$ is a von Neumann algebra if and only if $\mathcal{M}$ is closed under the weak operator topology, i.e., for all nets $A_{\alpha} \in \mathcal{M}$ and $A \in \mathcal{B}(\mathcal{H})$, if $\left\langle\psi, A_{\alpha} \psi\right\rangle \rightarrow\langle\psi, A \psi\rangle$ for all $\psi \in \mathcal{H}$ then $A \in \mathcal{M}$. We denote by $\mathcal{P}(\mathcal{M})$ the set of projections in a von Neumann algebra $\mathcal{M}$.

We say that $P$ and $Q$ are commuting, in symbols $P ! Q$, iff $[P, Q]=0$, where $[P, Q]=$ $P Q-Q P$. It is well-known that $P ! Q$ if and only if $P=(P \wedge Q) \vee\left(P \wedge Q^{\perp}\right)$. All the relations $P ! Q, Q ! P, P^{\perp} ! Q, P ! Q^{\perp}$, and $P^{\perp} ! Q^{\perp}$ are equivalent. For any subset $\mathcal{A} \subseteq \mathcal{Q}(\mathcal{H})$, we denote by $\mathcal{A}^{!}$the commutant of $\mathcal{A}$ in $\mathcal{Q}(\mathcal{H})$, i.e., $\mathcal{A}^{!}=\{P \in \mathcal{Q}(\mathcal{H}) \mid$ $P ! Q$ for all $Q \in \mathcal{A}\}$ [12, p. 23]. Then, $\mathcal{A}^{!}$is a complete orthomodular sublattice of $\mathcal{Q}(\mathcal{H})$, i.e., $\wedge \mathcal{S}, \vee \mathcal{S}, P^{\perp} \in \mathcal{A}^{!}$for any $\mathcal{S} \subseteq \mathcal{A}^{!}$and $P \in \mathcal{A}^{!}$. A logic on $\mathcal{H}$ is a subset $\mathcal{Q}$ of $\mathcal{Q}(\mathcal{H})$ satisfying $\mathcal{Q}=\mathcal{Q}^{! !}$. Thus, any logic on $\mathcal{H}$ is a complete orthomodular sublattice of $\mathcal{Q}(\mathcal{H})$. For any subset $\mathcal{A} \subseteq \mathcal{Q}(\mathcal{H})$, the smallest logic including $\mathcal{A}$ is the logic $\mathcal{A}^{! !}$called the logic generated by $\mathcal{A}$.

Then, we have

Proposition 2.1 A subset $\mathcal{Q} \subseteq \mathcal{Q}(\mathcal{H})$ is a logic on $\mathcal{H}$ if and only if $\mathcal{Q}=\mathcal{P}(\mathcal{M})$ for some von Neumann algebra $\mathcal{M}$ on $\mathcal{H}$.

Proof. Let $\mathcal{Q} \subseteq \mathcal{Q}(\mathcal{H})$ be such that $\mathcal{Q}^{!}=\mathcal{Q}$. Then, $\mathcal{Q}^{\prime}$ is a von Neumann algebra and obviously $\mathcal{Q}^{!}=\mathcal{Q}^{\prime} \cap \mathcal{Q}(\mathcal{H})=\mathcal{P}\left(\mathcal{Q}^{\prime}\right)$. Thus, we have $\mathcal{Q}=\mathcal{Q}^{! !}=\left(\mathcal{Q}^{!}\right)^{\prime} \cap \mathcal{Q}(\mathcal{H})=$ $\left(\mathcal{P}\left(\mathcal{Q}^{\prime}\right)\right)^{\prime} \cap \mathcal{Q}(\mathcal{H})=\mathcal{Q}^{\prime \prime} \cap \mathcal{Q}(\mathcal{H})=\mathcal{P}\left(Q^{\prime \prime}\right)$. Suppose $\mathcal{Q}=\mathcal{P}(\mathcal{M})$ for some von Neumann algebra $\mathcal{M}$ on $\mathcal{H}$. Then, we have $\mathcal{M}=\mathcal{Q}^{\prime \prime}$. Thus, we have $\mathcal{Q}^{!}=\mathcal{Q}^{\prime} \cap \mathcal{Q}(\mathcal{H})=\mathcal{P}\left(\mathcal{Q}^{\prime}\right)$ and $\mathcal{Q}^{! !}=\left[\mathcal{Q}^{\prime} \cap \mathcal{Q}(\mathcal{H})\right]^{\prime} \cap \mathcal{Q}(\mathcal{H})=\mathcal{Q}^{\prime \prime} \cap \mathcal{Q}(\mathcal{H})=\mathcal{P}\left(\mathcal{Q}^{\prime \prime}\right)=\mathcal{P}(\mathcal{M})$. Thus, we have $\mathcal{Q}^{! !}=\mathcal{Q}$. $Q E D$

We define the implication and the logical equivalence on $\mathcal{Q}$ by $P \rightarrow Q=P^{\perp} \vee(P \wedge Q)$ and $P \leftrightarrow Q=(P \rightarrow Q) \wedge(Q \rightarrow P)$. The following properties are useful in the later discussions. For the proofs, see e.g. Hardegree [9].

Proposition 2.2 Let $\mathcal{Q}$ be a logic on $\mathcal{H}$ and $P_{1}, P_{2}, P, Q \in \mathcal{Q}$. The following hold.

(i) $P \rightarrow Q=\mathcal{P}\{\psi \in \mathcal{H} \mid P \psi=(P \wedge Q) \psi\}$.

(ii) $P \rightarrow Q=\mathcal{P}\{\psi \in \mathcal{H} \mid P \psi \in \mathcal{R}(Q)\}$.

(iii) $P \leftrightarrow Q=\mathcal{P}\{\psi \in \mathcal{H} \mid P \psi=Q \psi\}$.

(iv) $Q \leq P_{1} \rightarrow P_{2}$ if and only if $\mathcal{P} \mathcal{R}\left(P_{1} Q\right) \leq P_{2}$.

(v) $\mathcal{P} \mathcal{R}(P Q)=P \wedge\left(P^{\perp} \vee Q\right)$.

A logic on $\mathcal{H}$ is called Boolean iff it is a Boolean algebra.

Proposition 2.3 The following assertions hold for any $\mathcal{A} \subseteq \mathcal{Q}(\mathcal{H})$.

(i) $\mathcal{A}$ is a Boolean logic if and only if $\mathcal{A}=\mathcal{A}^{! !} \subseteq \mathcal{A} !$.

(ii) If $\mathcal{A} \subseteq \mathcal{A}^{!}$, the subset $\mathcal{A}^{! !}$is the smallest Boolean logic including $\mathcal{A}$. 
Proof. To prove (i), suppose $\mathcal{A}=\mathcal{A}^{! !} \subseteq \mathcal{A}^{!}$. By the double commutation theorem, $\mathcal{A}^{\prime \prime}$ is the von Neumann algebra generated by $\mathcal{A}$. Since $\mathcal{A} \subseteq \mathcal{A}^{!}$, the von Neumann algebra $\mathcal{A}^{\prime \prime}$ is abelian, so that $\mathcal{A}=\mathcal{A}^{\prime !}=\mathcal{A}^{\prime \prime} \cap \mathcal{Q}(\mathcal{H})$ is a Boolean logic. Conversely, suppose that $\mathcal{A} \subseteq \mathcal{Q}(\mathcal{H})$ is a Boolean logic. Then, $P$ ! $Q$ for all $P, Q \in \mathcal{A}$, so that we have $\mathcal{A}=\mathcal{A}^{! !} \subseteq \mathcal{A}$ !, and assertion (i) follows. To prove (ii), suppose $\mathcal{A} \subseteq \mathcal{A}$ !. Then, $\mathcal{A} ! !$ is a Boolean sublogic including $\mathcal{A}$. Let $\mathcal{B}$ be a Boolean sublogic including $\mathcal{A}$. Then, we have $\mathcal{A}^{! !} \subseteq \mathcal{B}^{! !}=\mathcal{B}$. Thus, $\mathcal{A}^{! !}$is the smallest Boolean sublogic including $\mathcal{A}$. $Q E D$

The following proposition is useful in later discussions.

Proposition 2.4 Let $\mathcal{Q}$ be a logic on $\mathcal{H}$. The following hold.

(i) If $P_{\alpha} \in \mathcal{Q}$ and $P_{\alpha} ! Q$ for all $\alpha$, then $\left(\bigvee_{\alpha} P_{\alpha}\right) ! Q, \wedge_{\alpha} P_{\alpha} ! Q$, and $Q \wedge\left(\bigvee_{\alpha} P_{\alpha}\right)=$ $\vee_{\alpha}\left(Q \wedge P_{\alpha}\right)$.

(ii) If $P_{1}, P_{2} ! Q$, then $\left(P_{1} \rightarrow P_{2}\right) \wedge Q=\left[\left(P_{1} \wedge Q\right) \rightarrow\left(P_{2} \wedge Q\right)\right] \wedge Q$.

Proof. For the proof of (i), see Ref. [35. To prove (ii), let $P_{1}, P_{2} ! Q$. By the set theoretical definition of $\wedge$ and by Proposition 2.2 (ii), it suffices to show that $P_{1} \psi \in$ $\mathcal{R}\left(P_{2}\right)$ if and only if $\left(P_{1} \wedge Q\right) \psi \in \mathcal{R}\left(P_{2} \wedge Q\right)$ for every $\psi \in \mathcal{R}(Q)$. Let $\psi \in \mathcal{R}(Q)$. If $\left(P_{1} \wedge Q\right) \psi \in \mathcal{R}\left(P_{2} \wedge Q\right)$, we have $P_{1} \psi=P_{1} Q \psi=\left(P_{1} \wedge Q\right) \psi \in \mathcal{R}\left(P_{2} \wedge Q\right) \subseteq \mathcal{R}\left(P_{2}\right)$, so that $P_{1} \psi \in \mathcal{R}\left(P_{2}\right)$. Conversely, if $P_{1} \psi \in \mathcal{R}\left(P_{2}\right)$, we have $\left(P_{1} \wedge Q\right) \psi=P_{1} Q \psi=P_{1} \psi \in \mathcal{R}\left(P_{2}\right)$ and $\left(P_{1} \wedge Q\right) \psi=Q P_{1} \psi \in \mathcal{R}(Q)$, so that $\left(P_{1} \wedge Q\right) \psi \in \mathcal{R}\left(P_{2} \wedge Q\right)$. Thus, we have shown that (ii) holds. $Q E D$

Let $\mathcal{Q}$ be a logic on $\mathcal{H}$. Let $\mathcal{A} \subseteq \mathcal{Q}$. A Boolean subdomain of $\mathcal{A}$ in $\mathcal{Q}$ is any $E \in \mathcal{A}^{!} \cap \mathcal{Q}$ such that $P_{1} \wedge E$ ! $P_{2} \wedge E$ for all $P_{1}, P_{2} \in \mathcal{A}$. Denote by $S_{\mathcal{Q}}(\mathcal{A})$ the set of Boolean subdomains of $\mathcal{A}$ in $\mathcal{Q}$, i.e.,

$$
S_{\mathcal{Q}}(\mathcal{A})=\left\{E \in \mathcal{A}^{!} \cap \mathcal{Q} \mid P_{1} \wedge E ! P_{2} \wedge E \text { for all } P_{1}, P_{2} \in \mathcal{A}\right\} .
$$

We shall write $S_{\mathcal{Q}}\left(P_{1}, \cdots, P_{n}\right)=S_{\mathcal{Q}}\left(\left\{P_{1}, \cdots, P_{n}\right\}\right)$.

For any $E \in S_{\mathcal{Q}}(\mathcal{A})$, the logic generated by $\mathcal{A} \wedge E$ is a Boolean sublogic of $\mathcal{Q}$, since $(\mathcal{A} \wedge E)^{\prime \prime}$ is an abelian von Neumann algebra. The Boolean domain of $\mathcal{A}$ in $\mathcal{Q}$, denoted by $\Perp_{\mathcal{Q}}(\mathcal{A})$, is defined as the supremum of $S_{\mathcal{Q}}(\mathcal{A})$, i.e.,

$$
\Perp_{\mathcal{Q}}(\mathcal{A})=\bigvee S_{\mathcal{Q}}(\mathcal{A})
$$

By Proposition 2.4 (i) we have $\Perp_{\mathcal{Q}}(\mathcal{A}) \in \mathcal{A}^{!} \cap \mathcal{Q}$ and $P_{1} \wedge \Perp_{\mathcal{Q}}(\mathcal{A}) ! P_{2} \wedge \Perp_{\mathcal{Q}}(\mathcal{A})$ for all $P_{1}, P_{2} \in \mathcal{A}$, and hence

$$
\Perp_{\mathcal{Q}}(\mathcal{A})=\max \left\{E \in \mathcal{A}^{!} \cap \mathcal{Q} \mid P_{1} \wedge E ! P_{2} \wedge E \text { for all } P_{1}, P_{2} \in \mathcal{A}\right\} .
$$

See Ref. [35, p. 308] for the case $\mathcal{Q}=\mathcal{Q}(\mathcal{H})$.

Theorem 2.5 For any subset $\mathcal{A} \subseteq \mathcal{Q}$, we have

$$
\Perp_{\mathcal{Q}}(\mathcal{A})=\mathcal{P}\left\{\psi \in \mathcal{H} \mid\left[P_{1}, P_{2}\right] P_{3} \psi=0 \text { for all } P_{1}, P_{2}, P_{3} \in \mathcal{A}\right\},
$$

and $\Perp_{\mathcal{Q}}(\mathcal{A})=\Perp_{\mathcal{Q}(\mathcal{H})}(\mathcal{A})$. 
Proof. Let $F=\max \left\{E \in \mathcal{A}^{!} \mid P_{1} \wedge E\right.$ 。 $P_{2} \wedge E$ for all $\left.P_{1}, P_{2} \in \mathcal{A}\right\}$ and $G=\{\psi \in$ $\mathcal{H} \mid\left[P_{1}, P_{2}\right] P_{3} \psi=0$ for all $\left.P_{1}, P_{2}, P_{3} \in \mathcal{A}\right\}$. Let $\psi \in \mathcal{R}(F)$ and $P_{1}, P_{2}, P_{3} \in \mathcal{A}$. Then, the operators $P_{1} F, P_{2} F$, and $P_{3} F$ are mutually commuting. We have $\psi=F \psi$, and $P_{1} P_{2} P_{3} F=P_{1} F P_{2} F P_{3} F=P_{2} F P_{1} F P_{3} F=P_{2} P_{1} P_{3} F$. Hence, we obtain $P_{1} P_{2} P_{3} \psi=$ $P_{2} P_{1} P_{3} \psi$. Thus, $\psi \in G$ and $\mathcal{R}(F) \subseteq G$. Conversely, suppose $\psi \in G$. Let $M=(\mathcal{A} \psi)^{\perp \perp}$, the closed linear subspace spanned by $\mathcal{A} \psi$. Then, $M$ is invariant under all $P \in \mathcal{A}$, so that $\mathcal{P}(M) \in \mathcal{A}^{!}$. Let $N=\left\{\xi \in \mathcal{H} \mid\left[P_{1}, P_{2}\right] \xi=0\right.$ for all $\left.P_{1}, P_{2} \in \mathcal{A}\right\}$. Then, $N$ is a closed subspace such that $M \subseteq N$ by assumption, so that $\left[P_{1}, P_{2}\right] \mathcal{P}(M)=0$. Since $\mathcal{P}(M) \in \mathcal{A}^{!}$, we have $P_{1} \wedge \mathcal{P}(M)$ ! $P_{2} \wedge \mathcal{P}(M)$. Thus, we have $\psi \in M \subseteq \mathcal{R}(F)$ and $G \subseteq \mathcal{R}(F)$, so that we have shown the relation $\mathcal{R}(F)=G$. By the well-known relation $\mathcal{P} \mathcal{N}(X)=\mathcal{P} \mathcal{R}\left(X^{*}\right)^{\perp} \in \mathcal{Q}$, where $\mathcal{N}(X)=X^{-1}(0)$, for all $X \in \mathcal{Q}^{\prime \prime}$, we have $\mathcal{P}(G)=\bigwedge_{P_{1}, P_{2}, P_{3}} \mathcal{P} \mathcal{N}\left(\left[P_{1}, P_{2}\right] P_{3}\right) \in \mathcal{Q}$. It follows that $F \in \mathcal{Q}$, and hence we have $F=\Perp_{\mathcal{Q}}(\mathcal{A})$. Therefore, we have proved the relation $\Perp_{\mathcal{Q}}(\mathcal{A})=\mathcal{P}(G)$. The relation $\Perp_{\mathcal{Q}}(\mathcal{A})=\Perp_{\mathcal{Q}(\mathcal{H})}(\mathcal{A})$ now follows immediately. $Q E D$

Henceforth, for any logic $\mathcal{Q}$ on $\mathcal{H}$ and any subset $\mathcal{A} \subseteq \mathcal{Q}$, we abbreviate $\Perp(\mathcal{A})=$ $\Perp_{\mathcal{Q}}(\mathcal{A})$, which is the common element of $\mathcal{Q}(\mathcal{H})$ for any $\mathcal{Q}$ by the above theorem. A simpler characterization of the Boolean domain is obtained in terms of von Neumann algebra generated by $\mathcal{A}$ as follows.

Theorem 2.6 For any subset $\mathcal{A} \subseteq \mathcal{Q}$, we have

$$
\Perp(\mathcal{A})=\mathcal{P}\left\{\psi \in \mathcal{H} \mid[A, B] \psi=0 \text { for all } A, B \in \mathcal{A}^{\prime \prime}\right\} .
$$

Proof. Let $E=\mathcal{P}\left\{\psi \in \mathcal{H} \mid\left[P_{1}, P_{2}\right] P_{3} \psi=0\right.$ for all $\left.P_{1}, P_{2}, P_{3} \in \mathcal{A}\right\}$ and $F=\mathcal{P}\{\psi \in$ $\mathcal{H} \mid[A, B] \psi=0$ for all $\left.A, B \in \mathcal{A}^{\prime \prime}\right\}$. Suppose $\psi \in \mathcal{R}(F)$. Let $P_{1}, P_{2}, P_{3} \in \mathcal{A}$. We have $P_{2} P_{3} \in \mathcal{A}^{\prime \prime}$, and hence $P_{1}\left(P_{2} P_{3}\right) \psi=P_{2} P_{3} P_{1} \psi=P_{2} P_{1} P_{3} \psi$. It follows that $\psi \in \mathcal{R}(E)$. Since $E=\Perp(\mathcal{A})$, we have $E \in \mathcal{A}^{\prime}$. Let $P, Q \in \mathcal{A}$. We have $[P, Q E]=[P E, Q E]=$ $[P \wedge E, Q \wedge E]=0$. Since $P \in \mathcal{A}$ was arbitrary, we have $Q E \in \mathcal{A}^{\prime}$. Since $Q \in \mathcal{A}$ was arbitrary, we have $\mathcal{A}^{\prime \prime} E \subseteq \mathcal{A}^{\prime}$. Since $\mathcal{A}^{\prime}=\left(\mathcal{A}^{\prime \prime}\right)^{\prime}$, we have $X Y E=Y X E$ for any $X, Y \in \mathcal{A}^{\prime \prime}$. Thus, if $\psi \in \mathcal{R}(E)$, then we have $X Y \psi=Y X \psi$ and $\psi \in \mathcal{R}(F)$. Therefore, we conclude $F=\Perp(\mathcal{A})$. $Q E D$

\section{Universe of quantum sets}

We denote by $V$ the universe of sets which satisfies the Zermelo-Fraenkel set theory with the axiom of choice $(\mathrm{ZFC})$. Throughout this paper, we fix the language $\mathcal{L}(\in)$ for firstorder theory with equality having a binary relation symbol $\in$, bounded quantifier symbols $\forall x \in y, \exists x \in y$, and no constant symbols. For any class $U$, the language $\mathcal{L}(\in, U)$ is the one obtained by adding a name for each element of $U$. For convenience, we use the same symbol for an element of $U$ and its name in $\mathcal{L}(\in, U)$ as well as for the membership relation and the symbol $\in$.

To each statement $\phi$ of $\mathcal{L}(\in, U)$, the satisfaction relation $\langle U, \in\rangle \models \phi$ is defined by the following recursive rules:

1. $\langle U, \in\rangle \models u \in v \quad$ iff $\quad u \in v$.

2. $\langle U, \in\rangle \models u=v \quad$ iff $u=v$. 
3. $\langle U, \in\rangle \models \neg \phi \quad$ iff $\langle U, \in\rangle \models \phi$ does not hold.

4. $\langle U, \in\rangle \models \phi_{1} \wedge \phi_{2} \quad$ iff $\langle U, \in\rangle \models \phi_{1}$ and $\langle U, \in\rangle \models \phi_{2}$.

5. $\langle U, \in\rangle \models(\forall x) \phi(x)$ iff $\langle U, \in\rangle \models \phi(u)$ for all $u \in U$.

We regard the other logical connectives and quantifiers as defined symbols. Our assumption that $V$ satisfies ZFC means that if $\phi\left(x_{1}, \ldots, x_{n}\right)$ is provable in ZFC, i.e., $\mathrm{ZFC} \vdash \phi\left(x_{1}, \ldots, x_{n}\right)$, then $\langle V, \in\rangle \models \phi\left(u_{1}, \ldots, u_{n}\right)$ for any formula $\phi\left(x_{1}, \ldots, x_{n}\right)$ of $\mathcal{L}(\in)$ and all $u_{1}, \ldots, u_{n} \in V$.

Let $\mathcal{Q}$ be a logic on $\mathcal{H}$. For each ordinal $\alpha$, let

$$
V_{\alpha}^{(\mathcal{Q})}=\left\{u \mid u: \mathcal{D}(u) \rightarrow \mathcal{Q} \text { and } \mathcal{D}(u) \subseteq \bigcup_{\beta<\alpha} V_{\beta}^{(\mathcal{Q})}\right\}
$$

The $\mathcal{Q}$-valued universe $V^{(\mathcal{Q})}$ is defined by

$$
V^{(\mathcal{Q})}=\bigcup_{\alpha \in \mathrm{On}} V_{\alpha}^{(\mathcal{Q})}
$$

where On is the class of all ordinals. It is easy to see that $\mathcal{Q}_{1} \subseteq \mathcal{Q}_{2}$ if and only if $V_{\alpha}^{\left(\mathcal{Q}_{1}\right)} \subseteq V_{\alpha}^{\left(\mathcal{Q}_{2}\right)}$ for all $\alpha$. Thus, every $V^{(\mathcal{Q})}$ is a subclass of $V^{(\mathcal{Q}(\mathcal{H}))}$. For every $u \in V^{(\mathcal{Q})}$, the rank of $u$, denoted by $\operatorname{rank}(u)$, is defined as the least $\alpha$ such that $u \in V_{\alpha}^{(\mathcal{Q})}$. It is easy to see that if $u \in \mathcal{D}(v)$ then $\operatorname{rank}(u)<\operatorname{rank}(v)$

For $u \in V^{(\mathcal{Q})}$, we define the support of $u$, denoted by $L(u)$, by transfinite recursion on the rank of $u$ by the relation

$$
L(u)=\bigcup_{x \in \mathcal{D}(u)} L(x) \cup\{u(x) \mid x \in \mathcal{D}(u)\}
$$

For $\mathcal{A} \subseteq V^{(\mathcal{Q})}$ we write $L(\mathcal{A})=\bigcup_{u \in \mathcal{A}} L(u)$ and for $u_{1}, \ldots, u_{n} \in V^{(\mathcal{Q})}$ we write $L\left(u_{1}, \ldots, u_{n}\right)=$ $L\left(\left\{u_{1}, \ldots, u_{n}\right\}\right)$. Then, we obtain the following characterization of subuniverses of $V^{(\mathcal{Q}(\mathcal{H}))}$.

Proposition 3.1 Let $\mathcal{Q}$ be a logic on $\mathcal{H}$ and $\alpha$ an ordinal. For any $u \in V^{(\mathcal{Q}(\mathcal{H}))}$, we have $u \in V_{\alpha}^{(\mathcal{Q})}$ if and only if $u \in V_{\alpha}^{(\mathcal{Q}(\mathcal{H}))}$ and $L(u) \subseteq \mathcal{Q}$. In particular, $u \in V^{(\mathcal{Q})}$ if and only if $u \in V^{(\mathcal{Q}(\mathcal{H}))}$ and $L(u) \subseteq \mathcal{Q}$. Moreover, $\operatorname{rank}(u)$ is the least $\alpha$ such that $u \in V_{\alpha}^{(\mathcal{Q}(\mathcal{H}))}$ for any $u \in V^{(\mathcal{Q})}$.

Proof. Immediate from transfinite induction on $\alpha$. QED

Let $\mathcal{A} \subseteq V^{(\mathcal{Q})}$. The Boolean domain of $\mathcal{A}$, denoted by $\underline{\vee}(\mathcal{A})$, is defined by

$$
\underline{\vee}(\mathcal{A})=\Perp L(\mathcal{A})
$$

For any $u_{1}, \ldots, u_{n} \in V^{(\mathcal{Q})}$, we write $\underline{\vee}\left(u_{1}, \ldots, u_{n}\right)=\underline{\vee}\left(\left\{u_{1}, \ldots, u_{n}\right\}\right)$.

To each statement $\phi$ of $\mathcal{L}\left(\in, V^{(\mathcal{Q})}\right)$ we assign the $\mathcal{Q}$-valued truth value $\llbracket \phi \rrbracket_{\mathcal{Q}}$ by the following recursive rules:

1. $\llbracket u=v \rrbracket_{\mathcal{Q}}=\bigwedge_{u^{\prime} \in \mathcal{D}(u)}\left(u\left(u^{\prime}\right) \rightarrow \llbracket u^{\prime} \in v \rrbracket_{\mathcal{Q}}\right) \wedge \bigwedge_{v^{\prime} \in \mathcal{D}(v)}\left(v\left(v^{\prime}\right) \rightarrow \llbracket v^{\prime} \in u \rrbracket_{\mathcal{Q}}\right)$.

2. $\llbracket u \in v \rrbracket_{\mathcal{Q}}=\bigvee_{v^{\prime} \in \mathcal{D}(v)}\left(v\left(v^{\prime}\right) \wedge \llbracket u=v^{\prime} \rrbracket_{\mathcal{Q}}\right)$. 
3. $\llbracket \neg \phi \rrbracket_{\mathcal{Q}}=\llbracket \phi \rrbracket_{\mathcal{Q}}^{\perp}$.

4. $\llbracket \phi_{1} \wedge \phi_{2} \rrbracket_{\mathcal{Q}}=\llbracket \phi_{1} \rrbracket_{\mathcal{Q}} \wedge \llbracket \phi_{2} \rrbracket_{\mathcal{Q}}$.

5. $\llbracket \phi_{1} \vee \phi_{2} \rrbracket_{\mathcal{Q}}=\llbracket \phi_{1} \rrbracket_{\mathcal{Q}} \vee \llbracket \phi_{2} \rrbracket_{\mathcal{Q}}$.

6. $\llbracket \phi_{1} \rightarrow \phi_{2} \rrbracket_{\mathcal{Q}}=\llbracket \phi_{1} \rrbracket_{\mathcal{Q}} \rightarrow \llbracket \phi_{2} \rrbracket_{\mathcal{Q}}$.

7. $\llbracket \phi_{1} \leftrightarrow \phi_{2} \rrbracket_{\mathcal{Q}}=\llbracket \phi_{1} \rrbracket_{\mathcal{Q}} \leftrightarrow \llbracket \phi_{2} \rrbracket_{\mathcal{Q}}$.

8. $\llbracket(\forall x \in u) \phi(x) \rrbracket_{\mathcal{Q}}=\bigwedge_{u^{\prime} \in \mathcal{D}(u)}\left(u\left(u^{\prime}\right) \rightarrow \llbracket \phi\left(u^{\prime}\right) \rrbracket_{\mathcal{Q}}\right)$.

9. $\llbracket(\exists x \in u) \phi(x) \rrbracket_{\mathcal{Q}}=\bigvee_{u^{\prime} \in \mathcal{D}(u)}\left(u\left(u^{\prime}\right) \wedge \llbracket \phi\left(u^{\prime}\right) \rrbracket_{\mathcal{Q}}\right)$.

10. $\llbracket(\forall x) \phi(x) \rrbracket_{\mathcal{Q}}=\bigwedge_{u \in V^{(\mathcal{Q})}} \llbracket \phi(u) \rrbracket_{\mathcal{Q}}$.

11. $\llbracket(\exists x) \phi(x) \rrbracket_{\mathcal{Q}}=\bigvee_{\left.u \in V^{(\mathcal{Q})}\right)} \llbracket \phi(u) \rrbracket_{\mathcal{Q}}$.

We say that a statement $\phi$ of $\mathcal{L}\left(\in, V^{(\mathcal{Q})}\right)$ holds in $V^{(\mathcal{Q})}$ iff $\llbracket \phi \rrbracket_{\mathcal{Q}}=1$. A formula in $\mathcal{L}(\in)$ is called a $\Delta_{0}$-formula iff it has no unbounded quantifiers $\forall x$ or $\exists x$.

Theorem $3.2\left(\Delta_{0}\right.$-Absoluteness Principle) For any $\Delta_{0}$-formula $\phi\left(x_{1}, \ldots, x_{n}\right)$ of $\mathcal{L}(\in$ ) and $u_{1}, \ldots, u_{n} \in V^{(\mathcal{Q})}$, we have

$$
\llbracket \phi\left(u_{1}, \ldots, u_{n}\right) \rrbracket_{\mathcal{Q}}=\llbracket \phi\left(u_{1}, \ldots, u_{n}\right) \rrbracket_{\mathcal{Q}(\mathcal{H})} .
$$

Proof. The assertion is proved by the induction on the complexity of formulas and the rank of elements of $V^{(\mathcal{Q})}$. First, we shall prove by transfinite induction on $\alpha$ that (i) $\llbracket u=v \rrbracket_{\mathcal{Q}}=\llbracket u=v \rrbracket_{\mathcal{Q}(\mathcal{H})}$ for any $u, v \in V_{\alpha}^{(\mathcal{Q})}$, and (ii) $\llbracket u \in v \rrbracket_{\mathcal{Q}}=\llbracket u \in v \rrbracket_{\mathcal{Q}(\mathcal{H})}$ for any $u \in V_{\alpha}^{(\mathcal{Q})}$ and $v \in V_{\alpha+1}^{(\mathcal{Q})}$. If $\alpha=0$, relations (i) and (ii) trivially hold. To prove (i), let $u, v \in V_{\alpha}^{(\mathcal{Q})}$. By induction hypothesis on (ii), we have $\llbracket u^{\prime} \in v \rrbracket_{\mathcal{Q}}=\llbracket u^{\prime} \in v \rrbracket_{\mathcal{Q}(\mathcal{H})}$ and $\llbracket v^{\prime} \in u \rrbracket_{\mathcal{Q}}=\llbracket v^{\prime} \in u \rrbracket_{\mathcal{Q}(\mathcal{H})}$ for all $u^{\prime} \in \mathcal{D}(u)$ and $v^{\prime} \in \mathcal{D}(v)$. Thus, we have

$$
\begin{aligned}
\llbracket u=v \rrbracket_{\mathcal{Q}} & =\bigwedge_{u^{\prime} \in \mathcal{D}(u)}\left(u\left(u^{\prime}\right) \rightarrow \llbracket u^{\prime} \in v \rrbracket_{\mathcal{Q}}\right) \wedge \bigwedge_{v^{\prime} \in \mathcal{D}(v)}\left(v\left(v^{\prime}\right) \rightarrow \llbracket v^{\prime} \in u \rrbracket_{\mathcal{Q}}\right) \\
& =\bigwedge_{u^{\prime} \in \mathcal{D}(u)}\left(u\left(u^{\prime}\right) \rightarrow \llbracket u^{\prime} \in v \rrbracket_{\mathcal{Q}(\mathcal{H})}\right) \wedge \bigwedge_{v^{\prime} \in \mathcal{D}(v)}\left(v\left(v^{\prime}\right) \rightarrow \llbracket v^{\prime} \in u \rrbracket_{\mathcal{Q}(\mathcal{H})}\right) \\
& =\llbracket u=v \rrbracket_{\mathcal{Q}(\mathcal{H})} .
\end{aligned}
$$

To prove (ii), suppose $u \in V_{\alpha}^{(\mathcal{Q})}$ and $v \in V_{\alpha+1}^{(\mathcal{Q})}$. If $v^{\prime} \in \mathcal{D}(v)$, we have $v^{\prime} \in V_{\alpha}^{(\mathcal{Q})}$, and hence we have $\llbracket u=v^{\prime} \rrbracket_{\mathcal{Q}}=\llbracket u=v^{\prime} \rrbracket_{\mathcal{Q}(\mathcal{H})}$ from the above. Thus, we have

$$
\begin{aligned}
\llbracket u \in v \rrbracket_{\mathcal{Q}} & =\bigvee_{v^{\prime} \in \mathcal{D}(v)}\left(v\left(v^{\prime}\right) \wedge \llbracket u=v^{\prime} \rrbracket_{\mathcal{Q}}\right) \\
& =\bigvee_{v^{\prime} \in \mathcal{D}(v)}\left(v\left(v^{\prime}\right) \wedge \llbracket u=v^{\prime} \rrbracket_{\mathcal{Q}(\mathcal{H})}\right) \\
& =\llbracket u \in v \rrbracket_{\mathcal{Q}(\mathcal{H})} .
\end{aligned}
$$

Therefore, the assertion holds for atomic formulas. Any induction step adding a logical symbol works easily, even when bounded quantifiers are concerned, since the ranges of the supremum and the infimum are common for evaluating $\llbracket \cdots \rrbracket_{\mathcal{Q}}$ and $\llbracket \cdots \rrbracket_{\mathcal{Q}(\mathcal{H})}$. (This would not happen if we were to consider unbounded quantifiers.) $Q E D$ 
Henceforth, for any $\Delta_{0}$-formula $\phi\left(x_{1}, \ldots, x_{n}\right)$ and $u_{1}, \ldots, u_{n} \in V^{(\mathcal{Q})}$, we abbreviate $\llbracket \phi\left(u_{1}, \ldots, u_{n}\right) \rrbracket=\llbracket \phi\left(u_{1}, \ldots, u_{n}\right) \rrbracket_{\mathcal{Q}}$, which is the common $\mathcal{Q}$-valued truth value in all $V^{(\mathcal{Q})}$ such that $u_{1}, \ldots, u_{n} \in V^{(\mathcal{Q})}$.

The universe $V$ can be embedded in $V^{(\mathcal{Q})}$ by the following operation $\vee: v \mapsto \check{v}$ defined by the $\in$-recursion: for each $v \in V, \check{v}=\{\check{u} \mid u \in v\} \times\{1\}$. Then we have the following.

Theorem $3.3\left(\Delta_{0}\right.$-Elementary Equivalence Principle) For any $\Delta_{0}$ formula $\phi\left(x_{1}, \ldots, x_{n}\right)$ of $\mathcal{L}(\in)$ and $u_{1}, \ldots, u_{n} \in V$, we have $\langle V, \in\rangle \models$ $\phi\left(u_{1}, \ldots, u_{n}\right)$ if and only if $\llbracket \phi\left(\check{u}_{1}, \ldots, \check{u}_{n}\right) \rrbracket=1$.

Proof. Let $\mathbf{2}$ be the sublogic such that $\mathbf{2}=\{0,1\}$. Then, by induction it is easy to see that $\langle V, \in\rangle \models \phi\left(u_{1}, \ldots, u_{n}\right)$ if and only if $\llbracket \phi\left(\check{u}_{1}, \ldots, \check{u}_{n}\right) \rrbracket_{2}=1$ for any $\phi\left(x_{1}, \ldots, x_{n}\right)$ in $\mathcal{L}(\in)$, and this is equivalent to $\llbracket \phi\left(\check{u}_{1}, \ldots, \check{u}_{n}\right) \rrbracket=1$ for any $\Delta_{0}$-formula $\phi\left(x_{1}, \ldots, x_{n}\right)$ by the $\Delta_{0}$-Absoluteness Principle. QED

Takeuti 35] proved that the following modifications of the equality axioms hold for the case $\mathcal{Q}=\mathcal{Q}(\mathcal{H})$.

Theorem 3.4 Let $\mathcal{Q}$ be a logic on $\mathcal{H}$. For any $u, u^{\prime}, v, v^{\prime}, w \in V^{(\mathcal{Q})}$, we have the following.

(i) $\llbracket u=u \rrbracket=1$.

(ii) $\llbracket u=v \rrbracket=\llbracket v=u \rrbracket$.

(iii) $\underline{\vee}\left(u, v, u^{\prime}\right) \wedge \llbracket u=u^{\prime} \rrbracket \wedge \llbracket u \in v \rrbracket \leq \llbracket u^{\prime} \in v \rrbracket$.

(iv) $\underline{\vee}\left(u, v, u^{\prime}\right) \wedge \llbracket u \in v \rrbracket \wedge \llbracket v=v^{\prime} \rrbracket \leq \llbracket u \in v^{\prime} \rrbracket$.

(v) $\underline{\vee}(u, v, w) \wedge \llbracket u=v \rrbracket \wedge \llbracket v=w \rrbracket \leq \llbracket u=w \rrbracket$.

Proof. Takeuti 35] proved the assertions for the case $\mathcal{Q}=\mathcal{Q}(\mathcal{H})$, and the assertions for general $\mathcal{Q}$ follows from the $\Delta_{0}$-Absoluteness Principle. $Q E D$

Takeuti 35] gave examples in which the transitivity and the substitution laws do not hold without modifications, so that the relations

$$
\begin{aligned}
& \llbracket u=u^{\prime} \rrbracket \wedge \llbracket u \in v \rrbracket \leq \llbracket u^{\prime} \in v \rrbracket, \\
& \llbracket u \in v \rrbracket \wedge \llbracket v=v^{\prime} \rrbracket \leq \llbracket u \in v^{\prime} \rrbracket \\
& \llbracket u=v \rrbracket \wedge \llbracket v=w \rrbracket \leq \llbracket u=w \rrbracket
\end{aligned}
$$

do not hold in general.

Takeuti 35] introduced $n$-ary relation symbols $\underline{\vee}\left(x_{0}, \ldots, x_{n}\right)$ for any $n=2,3, \ldots$ in the language $\mathcal{L}(\in)$. We denote by $\mathcal{L}(\in, \underline{\vee})$ the language $\mathcal{L}(\epsilon)$ augmented by relation symbols $\underline{\vee}\left(x_{0}, \ldots, x_{n}\right)$. We extend the $\mathcal{Q}(\mathcal{H})$-valued truth value for all the statements in $\mathcal{L}\left(\in, \underline{\vee}, V^{(\mathcal{Q}(\mathcal{H}))}\right)$ by the relation

$$
\llbracket \underline{\vee}\left(x_{0}, \ldots, x_{n}\right) \rrbracket=\underline{\vee}\left(u_{0}, \ldots, u_{n}\right)
$$

for any $u_{0}, \ldots, u_{n} \in V^{(\mathcal{Q})}$.

Takeuti [35] showed that the following axioms and modifications of axioms of the ZFC holds in $V^{(\mathcal{Q}(\mathcal{H}))}$ :

Axiom of Infinity. $\llbracket \exists x \in \check{\omega}(x \in \check{\omega}) \wedge \forall x \in \check{\omega} \exists y \in \check{\omega}(x \in y) \rrbracket=1$. 
Axiom of Pair. $\underline{\vee}(u, v) \leq \llbracket \exists x(\underline{\vee}(u, v, x) \wedge \forall y(y \in x \leftrightarrow y=u \vee y=v))) \rrbracket$.

Axiom of Union. $\underline{\vee}(u) \leq \llbracket \exists v(\underline{\vee}(u, v) \wedge \forall x(\underline{\bigvee}(x, u) \rightarrow(x \in v \leftrightarrow \exists y \in u(x \in y)))) \rrbracket$.

Axiom of Replacement. $\llbracket \forall x \in u \exists y \phi(x, y) \rrbracket \leq \llbracket \exists v \forall x \in u \exists y \in v \phi(x, y) \rrbracket$.

Axiom of Power Set. $\underline{\vee}(u) \leq \llbracket \exists v(\underline{\vee}(u, v) \wedge \forall t(\underline{\vee}(u, v, t) \rightarrow(t \in v \leftrightarrow \forall x \in t(x \in u)))) \rrbracket$.

Axiom of Foundation. $\underline{\vee}(u) \wedge \llbracket \exists x \in u(x \in u) \rrbracket \leq \llbracket \exists x \in u \forall y \in u(\neg y \in u) \rrbracket$. $v)))$.

Axiom of Choice. $\underline{\vee}(u) \leq \llbracket \exists v(\underline{\vee}(u, v) \wedge \forall x \in u(\exists y \in x \exists ! z \in u(y \in z) \rightarrow \exists ! y \in x(y \in$

According to the above, Takeuti [35] concluded that a reasonable set theory holds in $V^{(\mathcal{Q}(\mathcal{H}))}$. However, it is still difficult to say what theorem holds in $V^{(\mathcal{Q}(\mathcal{H}))}$, since we have to construct the proof for each theorem using the above "axioms". It may be routine, but the above "axioms" do not ensure that we can tell what theorems hold without constructing proofs. In the next section, we shall solve this problem by establishing a unified transfer of theorems of ZFC to valid statements on $V^{(\mathcal{Q})}$.

\section{ZFC Transfer Principle in Quantum Set Theory}

Let $u \in V^{(\mathcal{Q})}$ and $p \in \mathcal{Q}$. The restriction $\left.u\right|_{p}$ of $u$ to $p$ is defined by the following transfinite recursion:

$$
\begin{aligned}
\mathcal{D}\left(\left.u\right|_{p}\right) & =\left\{\left.x\right|_{p} \mid x \in \mathcal{D}(u)\right\} \\
\left.u\right|_{p}\left(\left.x\right|_{p}\right) & =u(x) \wedge p
\end{aligned}
$$

for any $x \in \mathcal{D}(u)$. Note that our definition of restriction is simpler than the corresponding notion given by Takeuti [35], and we shall develop the theory of restriction along with a different line.

Proposition 4.1 For any $\mathcal{A} \subseteq V^{(\mathcal{Q})}$ and $p \in \mathcal{Q}$, we have

$$
L\left(\left\{\left.u\right|_{p} \mid u \in \mathcal{A}\right\}\right)=L(\mathcal{A}) \wedge p .
$$

Proof. By induction, it is easy to see the relation $L\left(\left.u\right|_{p}\right)=L(u) \wedge p$, so that the assertion follows easily. $Q E D$

Let $\mathcal{A} \subseteq V^{(\mathcal{Q})}$. The logic generated by $\mathcal{A}$, denoted by $\mathcal{Q}(\mathcal{A})$, is define by

$$
\mathcal{Q}(\mathcal{A})=L(\mathcal{A})^{! !}
$$

For $u_{1}, \ldots, u_{n} \in V^{(\mathcal{Q})}$, we write $\mathcal{Q}\left(u_{1}, \ldots, u_{n}\right)=\mathcal{Q}\left(\left\{u_{1}, \ldots, u_{n}\right\}\right)$.

Proposition 4.2 For any $\Delta_{0}$-formula $\phi\left(x_{1}, \ldots, x_{n}\right)$ in $\mathcal{L}(\in)$ and $u_{1}, \cdots, u_{n} \in V^{(\mathcal{Q}(\mathcal{H}))}$, we have $\llbracket \phi\left(u_{1}, \ldots, u_{n}\right) \rrbracket \in \mathcal{Q}\left(u_{1}, \ldots, u_{n}\right)$.

Proof. Let $\mathcal{A}=\left\{u_{1}, \ldots, u_{n}\right\}$. Since $L(\mathcal{A}) \subseteq \mathcal{Q}(\mathcal{A})$, it follows from Proposition 3.1 that $u_{1}, \ldots, u_{n} \in V^{\mathcal{Q}(\mathcal{A})}$. By the $\Delta_{0}$-Absoluteness Principle, we have $\llbracket \phi\left(u_{1}, \ldots, u_{n}\right) \rrbracket=$ $\llbracket \phi\left(u_{1}, \ldots, u_{n}\right) \rrbracket_{\mathcal{Q}(\mathcal{A})} \in \mathcal{Q}(\mathcal{A}) . Q E D$

Proposition 4.3 For any $\Delta_{0}$-formula $\phi\left(x_{1}, \ldots, x_{n}\right)$ of $\mathcal{L}(\epsilon)$ and $u_{1}, \ldots, u_{n} \in V^{(\mathcal{Q}(\mathcal{H}))}$, if

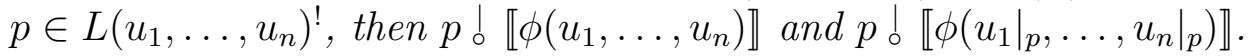


Proof. Let $u_{1}, \ldots, u_{n} \in V^{(\mathcal{Q})}$. If $p \in L\left(u_{1}, \ldots, u_{n}\right)^{!}$, then $p \in \mathcal{Q}\left(u_{1}, \ldots, u_{n}\right)^{!}$. From Proposition 4.2 $\llbracket \phi\left(u_{1}, \ldots, u_{n}\right) \rrbracket \in \mathcal{Q}\left(u_{1}, \ldots, u_{n}\right)$, so that $p$ ! $\llbracket \phi\left(u_{1}, \ldots, u_{n}\right) \rrbracket$. From Proposition 4.1, $L\left(\left.u_{1}\right|_{p}, \ldots,\left.u_{n}\right|_{p}\right)=L\left(u_{1}, \ldots, u_{n}\right) \wedge p$, and hence $p \in L\left(\left.u_{1}\right|_{p}, \ldots,\left.u_{n}\right|_{p}\right)^{!}$, so that $p ! \llbracket \phi\left(\left.u_{1}\right|_{p}, \ldots,\left.u_{n}\right|_{p}\right) \rrbracket . Q E D$

We define the binary relation $x_{1} \subseteq x_{2}$ by " $x_{1} \subseteq x_{2}$ " $=" \forall x \in x_{1}\left(x \in x_{2}\right)$." Then, by definition for any $u, v \in V^{(\mathcal{Q})}$ we have

$$
\llbracket u \subseteq v \rrbracket=\bigwedge_{u^{\prime} \in \mathcal{D}(u)} u\left(u^{\prime}\right) \rightarrow \llbracket u^{\prime} \in v \rrbracket,
$$

and we have $\llbracket u=v \rrbracket=\llbracket u \subseteq v \rrbracket \wedge \llbracket v \subseteq u \rrbracket$.

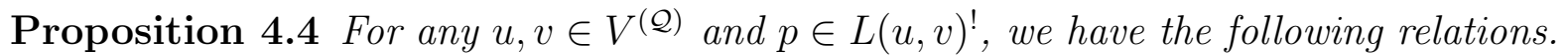

(i) $\left.\left.\llbracket u\right|_{p} \in v\right|_{p} \rrbracket=\llbracket u \in v \rrbracket \wedge p$.

(ii) $\left.\left.\llbracket u\right|_{p} \subseteq v\right|_{p} \rrbracket \wedge p=\llbracket u \subseteq v \rrbracket \wedge p$.

(iii) $\left.\llbracket u\right|_{p}=\left.v\right|_{p} \rrbracket \wedge p=\llbracket u=v \rrbracket \wedge p$.

Proof. We shall prove by transfinite induction on $\alpha$ that (i) holds for all $u \in V_{\alpha}^{(\mathcal{Q})}$ and $v \in V_{\alpha+1}^{(\mathcal{Q})}$ and that (ii) and (iii) hold for all $u, v \in V_{\alpha}^{(\mathcal{Q})}$. If $\alpha=0$, the relations trivially hold. To prove (ii), let $u, v \in V_{\alpha}^{(\mathcal{Q})}$ and $p \in L(u, v)^{!}$. Let $u^{\prime} \in \mathcal{D}(u)$. Since $L(u, v)^{!} \subseteq L\left(u^{\prime}, v\right)^{!}$, we have $p \in L\left(u^{\prime}, v\right)^{!}$. Then, we have $\left.\left.\llbracket u^{\prime}\right|_{p} \in v\right|_{p} \rrbracket=\llbracket u^{\prime} \in v \rrbracket \wedge p$ by induction hypothesis on (i). Thus, we have

$$
\begin{aligned}
\left.\left.\llbracket u\right|_{p} \subseteq v\right|_{p} \rrbracket & =\bigwedge_{u^{\prime} \in \mathcal{D}\left(\left.u\right|_{p}\right)}\left(\left.\left.u\right|_{p}\left(u^{\prime}\right) \rightarrow \llbracket u^{\prime} \in v\right|_{p} \rrbracket\right) \\
& =\bigwedge_{u^{\prime} \in \mathcal{D}(u)}\left(\left.\left.\left.u\right|_{p}\left(\left.u^{\prime}\right|_{p}\right) \rightarrow \llbracket u^{\prime}\right|_{p} \in v\right|_{p} \rrbracket\right) \\
& =\bigwedge_{u^{\prime} \in \mathcal{D}(u)}\left(u\left(u^{\prime}\right) \wedge p\right) \rightarrow\left(\llbracket u^{\prime} \in v \rrbracket \wedge p\right) .
\end{aligned}
$$

We have $p ! u\left(u^{\prime}\right)$ by assumption on $p$, and $p ! \llbracket u^{\prime} \in v \rrbracket$ by Proposition 4.3 so that $p ! u\left(u^{\prime}\right) \rightarrow \llbracket u^{\prime} \in v \rrbracket$ and $p !\left(u\left(u^{\prime}\right) \wedge p\right) \rightarrow\left(\llbracket u^{\prime} \in v \rrbracket \wedge p\right)$. From Proposition 2.4 (ii) we have

$$
p \wedge\left[\left(u\left(u^{\prime}\right) \wedge p\right) \rightarrow\left(\llbracket u^{\prime} \in v \rrbracket \wedge p\right)\right]=p \wedge\left(u\left(u^{\prime}\right) \rightarrow \llbracket u^{\prime} \in v \rrbracket\right) .
$$

Thus, from Proposition 2.4 (i) we have

$$
\begin{aligned}
\left.\left.p \wedge \llbracket u\right|_{p} \subseteq v\right|_{p} \rrbracket & =p \wedge \bigwedge_{u^{\prime} \in \mathcal{D}(u)}\left(u\left(u^{\prime}\right) \wedge p\right) \rightarrow\left(\llbracket u^{\prime} \in v \rrbracket \wedge p\right) \\
& =\bigwedge_{u^{\prime} \in \mathcal{D}(u)} p \wedge\left[\left(u\left(u^{\prime}\right) \wedge p\right) \rightarrow\left(\llbracket u^{\prime} \in v \rrbracket \wedge p\right)\right] \\
& =\bigwedge_{u^{\prime} \in \mathcal{D}(u)} p \wedge\left(u\left(u^{\prime}\right) \rightarrow \llbracket u^{\prime} \in v \rrbracket\right) \\
& =p \wedge \bigwedge_{u^{\prime} \in \mathcal{D}(u)}\left(u\left(u^{\prime}\right) \rightarrow \llbracket u^{\prime} \in v \rrbracket\right) \\
& =p \wedge \llbracket u \subseteq v \rrbracket .
\end{aligned}
$$


Thus, we have proved relation (ii) for all $u, v \in V_{\alpha}^{(\mathcal{Q})}$. Relation (iii) for all $u, v \in V_{\alpha}^{(\mathcal{Q})}$ follows easily from relation (ii). To prove (i), suppose $u \in V_{\alpha}^{(\mathcal{Q})}, v \in V_{\alpha+1}^{(\mathcal{Q})}$, and $p \in$ $L(u, v)^{!}$. Let $v^{\prime} \in \mathcal{D}(v)$. Since $L(u, v)^{!} \subseteq L\left(u, v^{\prime}\right)^{!}$, we have $p \in L\left(u, v^{\prime}\right)^{!}$. By relation (iii) for $u, v \in V_{\alpha}^{(\mathcal{Q})}$ shown above, we have $\left.\llbracket u\right|_{p}=\left.v^{\prime}\right|_{p} \rrbracket \wedge p=\llbracket u=v^{\prime} \rrbracket \wedge p$. By Proposition 4.3. we have $p ! \llbracket u=v^{\prime} \rrbracket$, so that $v\left(v^{\prime}\right), \llbracket u=v^{\prime} \rrbracket \in\{p\}^{!}$, and hence $p$ ! $v\left(v^{\prime}\right) \wedge \llbracket u=v^{\prime} \rrbracket$. Thus, we have

$$
\begin{aligned}
\left.\left.\llbracket u\right|_{p} \in v\right|_{p} \rrbracket & =\left.\left.\bigvee_{v^{\prime} \in \mathcal{D}\left(\left.v\right|_{p}\right)} v\right|_{p}\left(v^{\prime}\right) \wedge \llbracket u\right|_{p}=v^{\prime} \rrbracket \\
& =\left.\left.\bigvee_{v^{\prime} \in \mathcal{D}(v)} v\right|_{p}\left(\left.v^{\prime}\right|_{p}\right) \wedge \llbracket u\right|_{p}=\left.v^{\prime}\right|_{p} \rrbracket \\
& =\left.\bigvee_{v^{\prime} \in \mathcal{D}(v)} v\left(v^{\prime}\right) \wedge p \wedge \llbracket u\right|_{p}=\left.v^{\prime}\right|_{p} \rrbracket \\
& =\bigvee_{v^{\prime} \in \mathcal{D}(v)}\left(v\left(v^{\prime}\right) \wedge \llbracket u=v^{\prime} \rrbracket \wedge p\right) \\
& =\left(\bigvee_{v^{\prime} \in \mathcal{D}(v)} v\left(v^{\prime}\right) \wedge \llbracket u=v^{\prime} \rrbracket\right) \wedge p,
\end{aligned}
$$

where the last equality follows from Proposition 2.4 (i). Thus, by definition of $\llbracket u=v \rrbracket$ we obtain the relation $\left.\left.\llbracket u\right|_{p} \in v\right|_{p} \rrbracket=\llbracket u=v \rrbracket \wedge p$, and relation (i) for all $u \in V_{\alpha}^{(\mathcal{Q})}$ and $v \in V_{\alpha+1}^{(\mathcal{Q})}$ has been proved. Therefore, the assertion follows from transfinite induction on a. $Q E D$

Proposition 4.5 For any $\Delta_{0}$-formula $\phi\left(x_{1}, \ldots, x_{n}\right)$ of $\mathcal{L}(\in)$ and $u_{1}, \ldots, u_{n} \in V^{(\mathcal{Q})}$, if $p \in L\left(u_{1}, \ldots, u_{n}\right)^{!}$, then $\llbracket \phi\left(u_{1}, \ldots, u_{n}\right) \rrbracket \wedge p=\llbracket \phi\left(\left.u_{1}\right|_{p}, \ldots,\left.u_{n}\right|_{p}\right) \rrbracket \wedge p$.

Proof. We prove the assertion by induction on the complexity of $\phi\left(x_{1}, \ldots, x_{n}\right)$. From Proposition 4.4, the assertion holds for atomic formulas. Then, the verification of every induction step follows from the fact that the function $a \mapsto a \wedge p$ of all $a \in\{p\}^{!}$preserves all the supremum and infimum and satisfies $(a \rightarrow b) \wedge p=[(a \wedge p) \rightarrow(b \wedge p)] \wedge p$ from Proposition 2.4 (ii) and $a^{\perp} \wedge p=(a \wedge p)^{\perp} \wedge p$ for all $a, b \in\{p\}$ !. QED

Now, we can prove the following.

Theorem 4.6 (ZFC Transfer Principle) For any $\Delta_{0}$-formula $\phi\left(x_{1}, \ldots, x_{n}\right)$ of $\mathcal{L}(\in)$ and $u_{1}, \ldots, u_{n} \in V^{(\mathcal{Q})}$, if $\phi\left(x_{1}, \ldots, x_{n}\right)$ is provable in $Z F C$, then we have

$$
\underline{\vee}\left(u_{1}, \ldots, u_{n}\right) \leq \llbracket \phi\left(u_{1}, \ldots, u_{n}\right) \rrbracket .
$$

Proof. Let $p=\underline{\vee}\left(u_{1}, \ldots, u_{n}\right)$. Then, we have $a \wedge p ! b \wedge p$ for any $a, b \in L\left(u_{1}, \ldots, u_{n}\right)$, and hence there is a Boolean sublogic $\mathcal{B}$ such that $L\left(u_{1}, \ldots, u_{n}\right) \wedge p \subseteq \mathcal{B}$. From Proposition 4.1, we have $L\left(\left.u_{1}\right|_{p}, \ldots,\left.u_{n}\right|_{p}\right) \subseteq \mathcal{B}$. From Proposition 3.1, we have $\left.u_{1}\right|_{p}, \ldots,\left.u_{n}\right|_{p} \in V^{(\mathcal{B})}$. By the ZFC Transfer Principle of the Boolean valued universe [1, Theorem 1.33], we have $\llbracket \phi\left(\left.u_{1}\right|_{p}, \ldots,\left.u_{n}\right|_{p}\right) \rrbracket_{\mathcal{B}}=1$. By the $\Delta_{0}$-Absoluteness Principle, we have $\llbracket \phi\left(\left.u_{1}\right|_{p}, \ldots,\left.u_{n}\right|_{p}\right) \rrbracket=$ 1. From Proposition 4.5, we have $\llbracket \phi\left(u_{1}, \ldots, u_{n}\right) \rrbracket \wedge p=\llbracket \phi\left(\left.u_{1}\right|_{p}, \ldots,\left.u_{n}\right|_{p}\right) \rrbracket \wedge p=p$, and the assertion follows. $Q E D$ 


\section{Real numbers in quantum set theory}

Let $\mathbf{Q}$ be the set of rational numbers in $V$. We define the set of rational numbers in the model $V^{(\mathcal{Q})}$ to be $\mathbf{Q}$. We define a real number in the model by a Dedekind cut of the rational numbers. More precisely, we identify a real number with the upper segment of a Dedekind cut assuming that the lower segment has no end point. Therefore, the formal definition of the predicate $\mathbf{R}(x)$, " $x$ is a real number," is expressed by

$$
x \subseteq \check{\mathbf{Q}} \wedge \exists y \in \check{\mathbf{Q}}(y \in x) \wedge \exists y \in \check{\mathbf{Q}}(y \notin x) \wedge \forall y \in \check{\mathbf{Q}}(y \in x \leftrightarrow \forall z \in \check{\mathbf{Q}}(y<z \rightarrow z \in x)),
$$

where " $x \subseteq \check{\mathbf{Q}}$ " $=$ " $\forall y \in x(y \in \check{\mathbf{Q}})$." We define $\mathbf{R}^{(\mathcal{Q})}$ to be the interpretation of the set $\mathbf{R}$ of real numbers in $V^{(\mathcal{Q})}$ as follows.

$$
\mathbf{R}^{(\mathcal{Q})}=\left\{u \in V^{(\mathcal{Q})} \mid \mathcal{D}(u)=\mathcal{D}(\check{\mathbf{Q}}) \text { and } \llbracket \mathbf{R}(u) \rrbracket=1\right\} .
$$

Theorem 5.1 For any $u \in \mathbf{R}^{(\mathcal{Q})}$, we have the following.

(i) $u(\check{r})=\llbracket \check{r} \in u \rrbracket$ for all $r \in \mathbf{Q}$.

(ii) $\underline{\vee}(u)=1$.

Proof. Let $u \in \mathbf{R}^{(\mathcal{Q})}$ and $x \in \mathbf{Q}$. Then, we have

$$
\llbracket \check{x} \in u \rrbracket=\bigvee_{y \in \mathbf{Q}}(\llbracket \check{x}=\check{y} \rrbracket \wedge u(\check{y}))=u(\check{x}),
$$

and assertion (i) follows. We have

$$
\begin{aligned}
L(u) & =\bigcup_{s \in \mathcal{D}(u)} L(s) \cup\{u(s) \mid s \in \mathcal{D}(u)\} \\
& =\bigcup_{s \in \mathbf{Q}} L(\check{s}) \cup\{u(\check{s}) \mid s \in \mathbf{Q}\} \\
& =\{0,1, u(\check{s}) \mid s \in \mathbf{Q}\},
\end{aligned}
$$

so that it suffices to show that each $u(\check{s})$ with $s \in \mathbf{Q}$ is mutually commuting. By definition, we have

$$
\llbracket \forall y \in \check{\mathbf{Q}}(y \in u \leftrightarrow \forall z \in \check{\mathbf{Q}}(y<z \rightarrow z \in u)) \rrbracket=1 .
$$

Hence, we have

$$
u(\check{s})=\llbracket \check{s} \in u \rrbracket=\bigwedge_{s<t, t \in \mathbf{Q}} \llbracket \check{t} \in u \rrbracket .
$$

Thus, if $s_{1}<s_{2}$, then $u\left(\check{s}_{1}\right) \leq u\left(\check{s}_{2}\right)$, so that $u\left(\check{s}_{1}\right) ! u\left(\check{s}_{2}\right)$. Thus, each $u(\check{s})$ with $s \in \mathbf{Q}$ is mutually commuting, and assertion (ii) follows. $Q E D$

Proposition 5.2 If $\underline{\vee}(u) \wedge \llbracket \mathbf{R}(u) \rrbracket=1$, then there is a unique $v \in \mathbf{R}^{(\mathcal{Q})}$ such that $\underline{\vee}(u, v)=1$ and $\llbracket u=v \rrbracket=1$.

Proof. Let $\bar{u} \in V^{(\mathcal{Q})}$ be such that $\mathcal{D}(\bar{u})=\mathcal{D}(\check{Q})$ and $\bar{u}(\check{x})=\llbracket \check{x} \in u \rrbracket$ for all $x \in \mathbf{Q}$. Since $\underline{\vee}(u)=1$, there is a Boolean sublogic $\mathcal{B}$ such that $L(u) \subseteq L(u)^{!}=\mathcal{B}$. It is easy to see that $\check{Q}, \bar{u} \in V^{(\mathcal{B})}$ so that $\underline{\vee}(u, \bar{u})=1$, and $\llbracket \mathbf{R}(u) \rrbracket_{\mathcal{B}}=1$ by the $\Delta_{0}$-Absoluteness 
Principle. By definition, we have $\bar{u}(x) \rightarrow \llbracket x \in u \rrbracket=1$ for all $x \in \mathcal{D}(\bar{u})$, and hence $\llbracket \bar{u} \subseteq u \rrbracket=1$. On the other hand, we have $\llbracket u \subseteq \check{\mathbf{Q}}_{\rrbracket_{\mathcal{B}}}=1$ from $\llbracket \mathbf{R}(u) \rrbracket_{\mathcal{B}}=1$. Thus,

$$
\begin{aligned}
\llbracket u \subseteq \bar{u} \rrbracket & =\llbracket u \subseteq \bar{u} \rrbracket_{\mathcal{B}} \\
& =\llbracket \forall x(x \in u \rightarrow x \in \bar{u}) \rrbracket_{\mathcal{B}} \wedge \llbracket u \subseteq \check{\mathbf{Q}} \rrbracket_{\mathcal{B}} \\
& =\llbracket \forall x \in \check{Q}(x \in u \rightarrow x \in \bar{u}) \rrbracket_{\mathcal{B}} \\
& =\bigwedge_{x \in \mathcal{D}(\check{Q})}(\llbracket x \in u \rrbracket \rightarrow \llbracket x \in \bar{u} \rrbracket) \\
& =1 .
\end{aligned}
$$

To show the uniqueness, let $v_{1}, v_{2} \in \mathbf{R}^{(\mathcal{Q})}$ be such that $\llbracket u=v_{1} \rrbracket=\llbracket u=v_{2} \rrbracket=1$ and $\underline{\vee}\left(u, v_{1}\right)=\underline{\vee}\left(u, v_{2}\right)=1$. Let $x \in \mathbf{Q}$. Then, $\llbracket \check{x} \in v_{1} \rrbracket=\llbracket \check{x} \in u \rrbracket$ follows from $\llbracket u=v_{1} \rrbracket=\underline{\vee}\left(u, v_{1}, \check{x}\right)=1$, and we have $\llbracket \check{x} \in v_{2} \rrbracket=\llbracket \check{x} \in u \rrbracket$ similarly. Thus, we have $\llbracket \check{x} \in v_{1} \rrbracket=\llbracket \check{x} \in v_{2} \rrbracket$. Since $v_{1}(\check{x})=\llbracket \check{x} \in v_{1} \rrbracket$ and $v_{2}(\check{x})=\llbracket \check{x} \in v_{2} \rrbracket$, the relation $\llbracket v_{1}=v_{2} \rrbracket$ follows easily. $Q E D$

Theorem 5.3 If $\left(\mathbf{R}\left(x_{1}\right) \wedge \cdots \wedge \mathbf{R}\left(x_{n}\right)\right) \rightarrow \psi\left(x_{1}, \ldots, x_{n}, x_{n+1}, \ldots, x_{n+m}\right)$ is a $\Delta_{0}$-formula of $\mathcal{L}(\in)$ provable in $Z F C$, then for any $u_{1}, \ldots, u_{n} \in \mathbf{R}^{(\mathcal{Q})}$ and $u_{n+1}, \ldots, u_{n+m} \in V^{(\mathcal{Q})}$ we have

$$
\underline{\bigvee}\left(u_{1}, \ldots, u_{n+m}\right) \leq \llbracket \psi\left(u_{1}, \ldots, u_{n+m}\right) \rrbracket .
$$

Proof. By the ZFC transfer principle, we have

$$
\underline{\vee}\left(u_{1}, \ldots, u_{n+m}\right) \leq \llbracket \mathbf{R}\left(u_{1}\right) \wedge \cdots \wedge \mathbf{R}\left(u_{n}\right) \rrbracket \rightarrow \llbracket \psi\left(u_{1}, \ldots, u_{n+m}\right) \rrbracket,
$$

and the assertion follows from $\llbracket \mathbf{R}\left(u_{1}\right) \wedge \cdots \wedge \mathbf{R}\left(u_{n}\right) \rrbracket=1$ for any $u_{1}, \ldots, u_{n} \in \mathbf{R}^{(\mathcal{Q})}$. $Q E D$

In what follows, we write $r \wedge s=\min \{r, s\}$ and $r \vee s=\max \{r, s\}$ for any $r, s \in \mathbf{R}$. The Boolean domain $\underline{\vee}(u, v)$ of $u, v \in \mathbf{R}^{(\mathcal{Q})}$ is characterized as follows.

Theorem 5.4 For any $u, v \in \mathbf{R}^{(Q)}$, we have

$$
\underline{\vee}(u, v)=\mathcal{P}\{\psi \in \mathcal{H} \mid[u(\check{x}), v(\check{y})] \psi=0 \text { for all } x, y \in \mathbf{Q}\}
$$

Proof. We have $L(u, v)=\{0,1, u(\check{x}), v(\check{x}) \mid x \in \mathbf{Q}\}$. Let $\psi \in \mathcal{R} \underline{\bigvee}(u, v)$. From Theo$\operatorname{rem}[2.5$, we have $[u(\check{x}), v(\check{y})] u(\check{z}) \psi=0$ for all $x, y, z \in \mathbf{Q}$. Taking the limit $z \rightarrow \infty$ we have $[u(\check{x}), v(\check{y})] \psi=0$ for all $x, y \in \mathbf{Q}$. Conversely, suppose $[u(\check{x}), v(\check{y})] \psi=0$ for all $x, y \in \mathbf{Q}$. From Theorem 2.5, it suffices to show that $[u(\check{x}), v(\check{y})] u(\check{z}) \psi=0$ and $[u(\check{x}), v(\check{y})] v(\check{z}) \psi=0$ for any $x, y, z \in \mathbf{Q}$. We have $u(\check{x}) v(\check{y}) u(\check{z}) \psi=u(\check{x}) u(\check{z}) v(\check{y}) \psi=u(\check{x} \wedge \check{z}) v(\check{y}) \psi$, and $v(\check{y}) u(\check{x}) u(\check{z}) \psi=v(\check{y}) u(\check{x} \wedge \check{z}) \psi=u(\check{x} \wedge \check{z}) v(\check{y}) \psi$, so that $[u(\check{x}), v(\check{y})] u(\check{z}) \psi=0$. Similarly, we also have $[u(\check{x}), v(\check{y})] v(\check{z}) \psi=0$. This completes the proof. $Q E D$

The $\mathcal{Q}$-valued equality $\llbracket u=v \rrbracket$ for $u, v \in \mathbf{R}^{(\mathcal{Q})}$ is characterized as follows.

Theorem 5.5 For any $u, v \in \mathbf{R}^{(\mathcal{Q})}$ we have

$$
\llbracket u=v \rrbracket=\mathcal{P}\{\psi \in \mathcal{H} \mid \llbracket \check{r} \in u \rrbracket \psi=\llbracket \check{r} \in v \rrbracket \psi \text { for all } r \in \mathbf{Q}\} .
$$


Proof. From Theorem 5.2 (i) we have

$$
\begin{aligned}
\llbracket u=v \rrbracket & =\bigwedge_{r \in \mathbf{Q}}(u(\check{r}) \rightarrow \llbracket \check{r} \in v \rrbracket) \wedge \bigwedge_{r \in \mathbf{Q}}(v(\check{r}) \rightarrow \llbracket \check{r} \in u \rrbracket) \\
& =\bigwedge_{r \in \mathbf{Q}}(\llbracket \check{r} \in u \rrbracket \leftrightarrow \llbracket \check{r} \in v \rrbracket) .
\end{aligned}
$$

From Proposition 2.2 (iii), we have

$$
\llbracket \check{r} \in u \rrbracket \leftrightarrow \llbracket \check{r} \in v \rrbracket=\mathcal{P}\{\psi \in \mathcal{H} \mid \llbracket \check{r} \in u \rrbracket \psi=\llbracket \check{r} \in v \rrbracket \psi\}
$$

Thus, the assertion follows easily. $Q E D$

Theorem 5.6 For any $u, v \in \mathbf{R}^{(\mathcal{Q})}$ and $\psi \in \mathcal{H}$, the following conditions are all equivalent.

(i) $\psi \in \mathcal{R} \llbracket u=v \rrbracket$.

(ii) $u(\check{x}) \psi=v(\check{x}) \psi$ for any $x \in \mathbf{Q}$.

(iii) $u(\check{x}) v(\check{y}) \psi=v(\check{x} \wedge \check{y}) \psi$ for any $x, y \in \mathbf{Q}$.

(iv) $\langle u(\check{x}) \psi, v(\check{y}) \psi\rangle=\|v(\check{x} \wedge \check{y}) \psi\|^{2}$ for any $x, y \in \mathbf{Q}$.

Proof. The equivalence (i) $\Leftrightarrow$ (ii) follows from Theorem 5.5. Suppose (ii) holds. Then, we have $u(\check{x}) v(\check{y}) \psi=u(\check{x}) u(\check{y}) \psi=u(\check{x} \wedge \check{y}) \psi=v(\check{x} \wedge \check{y}) \psi$. Thus, the implication (ii) $\Rightarrow$ (iii) holds. Suppose (iii) holds. We have $\langle u(\check{x}) \psi, v(\check{y}) \psi\rangle=\langle\psi, u(\check{x}) v(\check{y}) \psi\rangle=\langle\psi, v(\check{x} \wedge \check{y}) \psi\rangle=$ $\|v(\check{x} \wedge \check{y}) \psi\|^{2}$, and hence the implication (iii) $\Rightarrow$ (iv) holds. Suppose (iv) holds. Then, we have $\langle u(\check{x}) \psi, v(\check{x}) \psi\rangle=\|v(\check{x}) \psi\|^{2}$ and $\langle v(\check{x}) \psi, u(\check{x}) \psi\rangle=\|u(\check{x}) \psi\|^{2}$. Consequently, we have $\|u(\check{x}) \psi-v(\check{x}) \psi\|^{2}=\|u(\check{x}) \psi\|^{2}+\|v(\check{x}) \psi\|^{2}-\langle u(\check{x}) \psi, v(\check{x}) \psi\rangle-\langle v(\check{x}) \psi, u(\check{x}) \psi\rangle=0$, and hence $u(\check{x}) \psi=v(\check{x}) \psi$. Thus, the implication (iv) $\Rightarrow$ (ii) holds, and the proof is completed. $Q E D$

The set $\mathbf{R}_{\mathcal{Q}}$ of real numbers in $V^{(\mathcal{Q})}$ is defined by

$$
\mathbf{R}_{\mathcal{Q}}=\mathbf{R}^{(\mathcal{Q})} \times\{1\} .
$$

The following theorem shows that the equality is an equivalence relation between real numbers in $V^{(\mathcal{Q})}$.

Theorem 5.7 The following relations hold in $V^{(\mathcal{Q})}$.

(i) $\llbracket\left(\forall u \in \mathbf{R}_{\mathcal{Q}}\right) u=u \rrbracket=1$.

(ii) $\llbracket\left(\forall u, v \in \mathbf{R}_{\mathcal{Q}}\right) u=v \rightarrow v=u \rrbracket=1$.

(iii) $\llbracket\left(\forall u, v, w \in \mathbf{R}_{\mathcal{Q}}\right) u=v \wedge v=w \rightarrow u=w \rrbracket=1$.

(iv) $\llbracket\left(\forall v \in \mathbf{R}_{\mathcal{Q}}\right)(\forall x, y \in v) x=y \wedge x \in v \rightarrow y \in v \rrbracket=1$.

(v) $\llbracket\left(\forall u, v \in \mathbf{R}_{\mathcal{Q}}\right)(\forall x \in u) x \in u \wedge u=v \rightarrow x \in v \rrbracket=1$.

Proof. Relations (i) and (ii) follow from Theorem 3.4. To prove (iii), let $u, v, w \in$ $\mathcal{D}\left(\mathbf{R}_{\mathcal{Q}}\right)=\mathbf{R}^{(\mathcal{Q})}$. Suppose $\psi \in \mathcal{R}(\llbracket u=v \rrbracket \wedge \llbracket v=w \rrbracket)$. Let $r \in \mathbf{Q}$. Then, we have $\llbracket \check{r} \in u \rrbracket \psi=\llbracket \check{r} \in v \rrbracket \psi$ and $\llbracket \check{r} \in v \rrbracket \psi=\llbracket \check{r} \in w \rrbracket \psi$ from Theorem [5.5. and hence $\llbracket \check{r} \in u \rrbracket \psi=$ $\llbracket \check{r} \in w \rrbracket \psi$. Since $r$ was arbitrary, we obtain $\psi \in \mathcal{R} \llbracket u=w \rrbracket$. Thus, we have

$$
\bigwedge_{u, v, w \in \mathcal{D}\left(\mathbf{R}_{\mathcal{Q}}\right)} \llbracket u=v \wedge v=w \rightarrow u=w \rrbracket=1,
$$


and relation (iii) holds. To prove (iv), let $v \in \mathbf{R}^{(\mathcal{Q})}$, and let $x, y \in \mathcal{D}(v)$. Then, we have $s, t \in \mathbf{Q}$ such that $x=\check{s}$ and $y=\check{t}$. If $s \neq t$, then $\llbracket x=y \rrbracket=0$, and the relation trivially holds. If $s=t$, then we have $\llbracket x \in v \rrbracket=\llbracket \check{s} \in v \rrbracket=\llbracket \check{t} \in v \rrbracket=\llbracket y \in v \rrbracket$, and hence (iv) holds. To probe $(\mathrm{v})$, let $u, v \in \mathbf{R}^{(\mathcal{Q})}$, and let $x \in \mathcal{D}(u)$. Suppose $\psi \in \mathcal{R}(\llbracket x \in u \rrbracket \wedge \llbracket u=v \rrbracket)$. Let $r \in \mathbf{Q}$ such that $x=\check{r}$. Then, $\llbracket \check{r} \in u \rrbracket \psi=\psi$ and $\llbracket \check{r} \in u \rrbracket \psi=\llbracket \check{r} \in v \rrbracket \psi$ from Theorem 5.5. and hence $\llbracket x \in v \rrbracket \psi=\psi$, so that $\psi \in \mathcal{R} \llbracket x \in v \rrbracket$. Thus, (v) holds. $Q E D$

The following theorem shows commutativity follows from equality in $\mathbf{R}^{(\mathcal{Q})}$.

Theorem 5.8 For any $u_{1}, \ldots, u_{n} \in \mathbf{R}^{(\mathcal{Q})}$, we have

$$
\llbracket u_{1}=u_{2} \wedge \cdots \wedge u_{n-1}=u_{n} \rrbracket \leq \underline{\vee}\left(u_{1}, \ldots, u_{n}\right) .
$$

Proof. We have $L\left(u_{1}, \ldots, u_{n}\right)=\left\{0,1, u_{1}(\check{x}), \ldots, u_{n}(\check{x}) \mid x \in \mathcal{Q}\right\}$. Let $\psi \in \mathcal{R} \llbracket u_{1}=$ $u_{2} \wedge \cdots \wedge u_{n-1}=u_{n} \rrbracket$. From Theorem [2.5, it suffices to show that $u_{i}(\check{x}) u_{j}(\check{y}) u_{k}(\check{z}) \psi=$ $u_{j}(\check{y}) u_{i}(\check{x}) u_{k}(\check{z}) \psi$ for any $i, j, k=1, \ldots, n$ and $x, y, z \in \mathbf{Q}$. From Theorem 5.7 (iii), we have $\psi \in \mathcal{R} \llbracket u_{j}=u_{k} \rrbracket \cap \mathcal{R} \llbracket u_{i}=u_{k} \rrbracket$. From Theorem 5.6, we have $u_{i}(\check{x}) u_{j}(\check{y}) u_{k}(\check{z}) \psi=$ $u_{i}(\check{x}) u_{k}(\check{y} \wedge \check{z}) \psi=u_{k}(\check{x} \wedge \check{y} \wedge \check{z}) \psi$ and $u_{j}(\check{y}) u_{i}(\check{x}) u_{k}(\check{z}) \psi=u_{j}(\check{y}) u_{k}(\check{x} \wedge \check{z}) \psi=u_{k}(\check{x} \wedge \check{y} \wedge \check{z}) \psi$. Thus, the above relation follows easily. $Q E D$

The following theorem shows that the equality between real numbers in $V^{(\mathcal{Q})}$ satisfies the substitution law for $\Delta_{0}$-formulas.

Theorem $5.9\left(\Delta_{0}\right.$-Substitution Law) For any $\Delta_{0}$-formula $\phi\left(x_{1}, \ldots, x_{n}\right)$ in $\mathcal{L}(\in)$, we have

$$
\begin{aligned}
& \llbracket\left(\forall u_{1}, \ldots, u_{n}, v_{1}, \ldots, v_{n}\right.\left.\in \mathbf{R}_{\mathcal{Q}}\right) \\
&\left(u_{1}=v_{1} \wedge \cdots \wedge u_{n}=v_{n}\right) \rightarrow\left(\phi\left(u_{1}, \ldots, u_{n}\right) \leftrightarrow \phi\left(v_{1}, \ldots, v_{n}\right)\right) \rrbracket=1
\end{aligned}
$$

Proof. Let $u_{1}, \ldots, u_{n}, v_{1}, \ldots, v_{n} \in \mathbf{R}^{(\mathcal{Q})}$. From the ZFC Transfer Principle, we have

$$
\underline{\vee}\left(u_{1}, \ldots, u_{n}, v_{1}, \ldots, v_{n}\right) \leq \llbracket u_{1}=v_{1} \wedge \cdots \wedge u_{n}=v_{n} \rrbracket \rightarrow \llbracket \phi\left(u_{1}, \ldots, u_{n}\right) \leftrightarrow \phi\left(v_{1}, \ldots, v_{n}\right) \rrbracket .
$$

Since $\underline{\vee}\left(u_{1}, \ldots, u_{n}, v_{1}, \ldots, v_{n}\right) \in L\left(u_{1}, v_{1}, \ldots, u_{n}, v_{n}\right)^{!}$, we have

$$
\llbracket u_{1}=v_{1} \wedge \cdots \wedge u_{n}=v_{n} \rrbracket \wedge \underline{\vee}\left(u_{1}, \ldots, u_{n}, v_{1}, \ldots, v_{n}\right) \leq \llbracket \phi\left(u_{1}, \ldots, u_{n}\right) \leftrightarrow \phi\left(v_{1}, \ldots, v_{n}\right) \rrbracket .
$$

From Theorem 5.8, we have

$$
\llbracket u_{1}=v_{1} \wedge \cdots \wedge u_{n}=v_{n} \rrbracket=\llbracket u_{1}=v_{1} \wedge \cdots \wedge u_{n}=v_{n} \rrbracket \wedge \underline{\vee}\left(u_{1}, \ldots, u_{n}, v_{1}, \ldots, v_{n}\right) .
$$

Thus, we have

$$
\bigwedge_{u_{1}, \ldots, u_{n}, v_{1}, \ldots, v_{n} \in \mathcal{D}\left(\mathbf{R}_{\mathcal{Q}}\right)} \llbracket\left(u_{1}=v_{1} \wedge \cdots \wedge u_{n}=v_{n}\right) \rightarrow\left(\phi\left(u_{1}, \ldots, u_{n}\right) \leftrightarrow \phi\left(v_{1}, \ldots, v_{n}\right)\right) \rrbracket=1,
$$

and the assertion follows. $Q E D$ 
Corollary 5.10 For any $\Delta_{0}$-formula $\phi\left(x_{1}, \ldots, x_{n}\right)$ in $\mathcal{L}(\in)$ and any $u_{1}, \ldots, u_{n}, v_{1}, \ldots, v_{n} \in \mathbf{R}_{\mathcal{Q}}$, we have

$$
\llbracket u_{1}=v_{1} \wedge \cdots \wedge u_{n}=v_{n} \rrbracket \wedge \llbracket \phi\left(u_{1}, \ldots, u_{n}\right) \rrbracket \leq \llbracket \phi\left(v_{1}, \ldots, v_{n}\right) \rrbracket .
$$

Proof. From Theorem 5.9, we have

$$
\llbracket u_{1}=v_{1} \wedge \cdots \wedge u_{n}=v_{n} \rrbracket \leq \llbracket \phi\left(u_{1}, \ldots, u_{n}\right) \rrbracket \rightarrow \llbracket \phi\left(v_{1}, \ldots, v_{n}\right) \rrbracket .
$$

Thus, Proposition 2.2 (iv) leads to

$$
\llbracket u_{1}=v_{1} \wedge \cdots \wedge u_{n}=v_{n} \rrbracket \wedge \llbracket \phi\left(u_{1}, \ldots, u_{n}\right) \rrbracket \leq \llbracket \phi\left(v_{1}, \ldots, v_{n}\right) \rrbracket .
$$

$Q E D$

For any $u, v \in \mathbf{R}^{(\mathcal{Q})}, u \leq v, u<v$, and $u<v \leq w$ are $\Delta_{0}$-formula such that

$$
\begin{aligned}
" u \leq v " & =" v \subseteq u ", \\
" u<v " & ="(u \leq v) \wedge \neg(u=v) ", \\
" u<v \leq w " & ="(u<v) \wedge(v \leq w) .
\end{aligned}
$$

Recall that for any $r \in R$ the embedding $\check{r} \in V^{(\mathcal{Q})}$ satisfies

$$
\mathcal{D}(\check{r})=\{\check{x} \mid r \leq x \in \mathbf{Q}\} \quad \text { and } \quad \check{r}(\check{x})=1
$$

for all $x \in \mathbf{Q}$ with $r \leq x$. In order to make the counter part of $r \in \mathbf{R}$ in $\mathbf{R}^{(\mathcal{Q})}$, for any $r \in R$, we define $\tilde{r} \in \mathbf{R}^{(\mathcal{Q})}$ by

$$
\mathcal{D}(\tilde{r})=\mathcal{D}(\check{\mathbf{Q}}) \quad \text { and } \quad \tilde{r}(\check{t})=\llbracket \check{r} \leq \check{t} \rrbracket
$$

for all $t \in \mathbf{Q}$.

Proposition 5.11 Let $r \in \mathbf{Q}, s, t \in \mathbf{R}$, and $u \in \mathbf{R}^{(\mathcal{Q})}$. We have the following relations.

(i) $\llbracket \check{r} \in \tilde{s} \rrbracket=\llbracket \check{s} \leq \check{r} \rrbracket$.

(ii) $\llbracket \tilde{s} \leq \tilde{t} \rrbracket=\llbracket \check{s} \leq \check{t} \rrbracket$.

(iii) $\llbracket u \leq \tilde{t} \rrbracket=\bigwedge_{t<x \in \mathbf{Q}} u(\check{x})$.

Proof. We have

$$
\llbracket \check{r} \in \tilde{s} \rrbracket=\bigvee_{x \in \mathcal{D}(\tilde{s})} \llbracket \check{r}=x \rrbracket \wedge \tilde{s}(x)=\bigvee_{x \in \mathbf{Q}} \llbracket \check{r}=\check{x} \rrbracket \wedge \tilde{s}(\check{x})=\tilde{s}(\check{r})=\llbracket \check{s} \leq \check{r} \rrbracket,
$$

so that (i) holds. We have

$$
\llbracket \tilde{s} \leq \tilde{t} \rrbracket=\llbracket(\forall x \in \tilde{t}) x \in \tilde{s} \rrbracket=\bigwedge_{x \in \mathbf{Q}} \llbracket \check{t} \leq \check{x} \rrbracket \rightarrow \llbracket \check{s} \leq \check{x} \rrbracket=\bigwedge_{t \leq x \in \mathbf{Q}} \llbracket \check{s} \leq \check{x} \rrbracket=\llbracket \check{s} \leq \check{t} \rrbracket,
$$

and hence (ii) holds. We have

$$
\llbracket u \leq \tilde{t} \rrbracket=\llbracket(\forall x \in \tilde{t}) x \in u \rrbracket=\bigwedge_{x \in \mathbf{Q}} \llbracket \check{t} \leq \check{x} \rrbracket \rightarrow u(\check{x})=\bigwedge_{t \leq x \in \mathbf{Q}} u(\check{x}),
$$

so that (iii) holds. QED 


\section{Applications to operator theory and quantum mechanics}

Let $\mathcal{M}$ be a von Neumann algebra on a Hilbert space $\mathcal{H}$ and let $\mathcal{Q}$ be a logic of projections in $\mathcal{M}$. Then, $\mathcal{M}=\mathcal{Q}^{\prime \prime}$ and every von Neumann algebra arises in this way from a logic $\mathcal{Q}$ on $\mathcal{H}$. A closed operator $A$ (densely defined) on $\mathcal{H}$ is said to be affiliated with $\mathcal{M}$, in symbols $A \eta \mathcal{M}$, iff $U^{*} A U=A$ for any unitary operator $U \in \mathcal{M}^{\prime}$. Let $A$ be a self-adjoint operator (densely defined) on $\mathcal{H}$ and let $A=\int_{\mathbf{R}} \lambda d E^{A}(\lambda)$ be its spectral decomposition, where $\left\{E^{A}(\lambda)\right\}_{\lambda \in \mathbf{R}}$ is the resolution of the identity belonging to $A$. It is well-known that $A \eta \mathcal{Q}^{\prime \prime}$ if and only if $E^{A}(\lambda) \in \mathcal{Q}$ for every $\lambda \in \mathbf{R}$. Denote by $\overline{\mathcal{M}}_{S A}$ the set of self-adjoint operators affiliated with $\mathcal{M}$. Two self-adjoint operators $A$ and $B$ are said to commute, in symbols $A ! B$, iff $E^{A}(\lambda) ! E^{B}\left(\lambda^{\prime}\right)$ for every pair $\lambda, \lambda^{\prime}$ of reals.

Let $\mathcal{B}$ be a Boolean logic on $\mathcal{H}$. Takeuti [32] showed that there is a one-to-one correspondence between $\mathbf{R}^{(\mathcal{B})}$ and ${\overline{\left(\mathcal{B}^{\prime \prime}\right)}}_{S A}$ as follows. Let $u \in \mathbf{R}^{(\mathcal{B})}$. Then, we have $u(\check{r}) \in \mathcal{B}$ for all $r \in \mathbf{Q}$ and the following are easily checked.

(i) $\bigwedge_{r \in \mathbf{Q}} u(\check{r})=0$.

(ii) $\bigvee_{r \in \mathbf{Q}} u(\check{r})=1$.

(iii) $u(\check{r})=\bigwedge_{r<s \in \mathbf{Q}} u(\check{s})$ for every $r \in \mathbf{Q}$.

In fact, (i) follows from $\llbracket \exists y \in \check{\mathbf{Q}}(y \notin u) \rrbracket=1$, (ii) follows from $\llbracket \exists y \in \mathbf{Q}(y \in u) \rrbracket=1$, and (iii) follows from $\llbracket \forall y \in \grave{\mathbf{Q}}(y \in u \leftrightarrow \forall z \in \grave{\mathbf{Q}}(y<z \rightarrow z \in u)) \rrbracket=1$.

For any $u \in \mathbf{R}^{(\mathcal{B})}$ and $\lambda \in \mathbf{R}$, we define $E^{u}(\lambda)$ by

$$
E^{u}(\lambda)=\bigwedge_{\lambda<r \in \mathbf{Q}} u(\check{r})
$$

Then, we have the following.

(i) $\bigwedge_{r \in \mathbf{Q}} E^{u}(\lambda)=0$

(ii) $\bigvee_{r \in \mathbf{Q}} E^{u}(\lambda)=1$.

(iii) $E^{u}(\lambda)=\bigwedge_{\lambda<\mu} E^{u}(\mu)$ for every $\lambda \in \mathbf{Q}$.

The above relations show that $\left\{E^{u}(\lambda)\right\}_{\lambda \in \mathbf{R}}$ is a resolution of the identity in $\mathcal{B}$ and hence by the spectral theorem there is a self-adjoint operator $\hat{u} \eta \mathcal{B}^{\prime \prime}$ uniquely satisfying $\hat{u}=$ $\int_{\mathbf{R}} \lambda d E^{u}(\lambda)$. On the other hand, let $A \eta \mathcal{B}^{\prime \prime}$ be a self-adjoint operator. We define $\tilde{A} \in V^{(\mathcal{B})}$ by

$$
\mathcal{D}(\tilde{A})=\mathcal{D}(\check{\mathbf{Q}}) \text { and } \tilde{A}(\check{r})=E^{A}(r) \text { for all } r \in \mathbf{Q} .
$$

Then, it is easy to see that $\tilde{A} \in \mathbf{R}^{(\mathcal{B})}$ and we have $(\hat{u})^{r}=u$ for all $u \in \mathbf{R}^{(\mathcal{B})}$ and $(\tilde{A})^{r}=A$ for all $A \in{\overline{\left(\mathcal{B}^{\prime \prime}\right)}}_{S A}$. Therefore, the correspondence between $\mathbf{R}^{(\mathcal{B})}$ and ${\overline{\left(\mathcal{B}^{\prime \prime}\right)}}_{S A}$ is a one-to-one correspondence. We call the above correspondence the Takeuti correspondence. It should be noted that for any $a \in \mathbf{R}$, the real $\tilde{a} \in \mathbf{R}^{(\mathcal{Q})}$ corresponds to the scalar operator $a 1$ under the Takeuti correspondence.

Now, we have the following.

Theorem 6.1 Let $\mathcal{Q}$ be a logic on $\mathcal{H}$. The relations 
(i) $E^{A}(\lambda)=\bigwedge_{\lambda<r \in \mathbf{Q}} u(\check{r})$ for all $\lambda \in \mathbf{Q}$,

(ii) $u(\check{r})=E^{A}(r)$ for all $r \in \mathbf{Q}$,

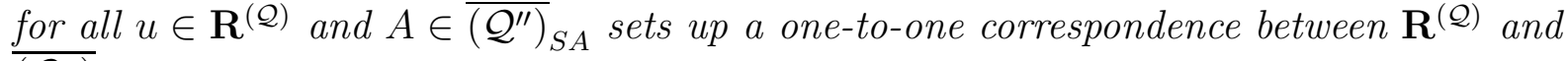
${\overline{\left(\mathcal{Q}^{\prime \prime}\right)}}_{S A}$.

Proof. Let $u \in \mathbf{R}^{(\mathcal{Q})}$. From Theorem 5.1 we have $\underline{\vee}(u)=1$. Thus, the logic $L(u)^{! !}$ generated by $L(u)$ is a Boolean logic. Let $\mathcal{B}=L(u)^{! !}$. By the $\Delta_{0}$-Absoluteness Principle, $u \in \mathbf{R}^{(\mathcal{B})}$. Thus, by Takeuti's result above there is a self-adjoint operator $A=\hat{u} \eta \mathcal{B}^{\prime \prime}$ satisfying relations (i) and (ii). Since $\mathcal{B}^{\prime \prime} \subseteq \mathcal{Q}^{\prime \prime}$, we have shown that for any $u \in \mathbf{R}^{(\mathcal{Q})}$ there exists $A \eta \mathcal{Q}^{\prime \prime}$ satisfying (i) and (ii), and the uniqueness of such $A$ follows easily. On the other hand, let $A \eta \mathcal{Q}^{\prime \prime}$. Let $\mathcal{B}$ be the Boolean logic generated by $\left\{E^{A}(\lambda)\right\}_{\lambda \in \mathbf{R}}$. Then, by Takeuti's result above we have $u=\tilde{A} \in V^{(\mathcal{B})}$ satisfying relations (i) and (ii). Since $A \eta \mathcal{Q}^{\prime \prime}$, we have $\mathcal{B} \subseteq \mathcal{P}\left(\mathcal{Q}^{\prime \prime}\right)=\mathcal{Q}$, and hence $u \in V^{(\mathcal{Q})}$. By the $\Delta_{0}$-Absoluteness Principle, we also have $u \in \mathbf{R}^{(\mathcal{Q})}$. Thus, we have proved that relations (i) and (ii) determine a one-to-one correspondence between $\mathbf{R}^{(\mathcal{Q})}$ and ${\overline{\left(\mathcal{Q}^{\prime \prime}\right)}}_{S A}$. $Q E D$

Let $E^{A}(\lambda)$ be the resolution of the identity belonging to a self-adjoint operator $A$. Let $a<b \in \mathbf{R}$. For the interval $I=(a, b]$, we define

$$
E^{A}(I)=E^{A}(b)-E^{A}(a),
$$

and we define the corresponding interval $\tilde{I}$ of real numbers in $V^{(\mathcal{Q})}$ by

$$
\mathcal{D}(\tilde{I})=\mathbf{R}^{(\mathcal{Q})} \quad \text { and } \quad \tilde{I}(u)=\llbracket \tilde{a}<u \rrbracket \wedge \llbracket u \leq \tilde{b} \rrbracket
$$

for all $u \in \mathbf{R}^{(\mathcal{Q})}$.

Theorem 6.2 Let $\mathcal{Q}$ be a logic on $\mathcal{H}$. For any self-adjoint operator $A \eta \mathcal{Q}^{\prime \prime}$ and any interval $I=(a, b]$, we have

$$
\llbracket \tilde{A} \in \tilde{I} \rrbracket=E^{A}(I)
$$

Proof. Let $u \in \mathbf{R}^{(\mathcal{Q})}$ and $I \in(a, b]$. We have

$$
\llbracket u \in \tilde{I} \rrbracket=\bigvee_{v^{\prime} \in \mathbf{R}^{(\mathcal{Q})}} \llbracket \tilde{a}<v^{\prime} \rrbracket \wedge \llbracket v^{\prime} \leq \tilde{b} \rrbracket \wedge \llbracket u=v^{\prime} \rrbracket .
$$

From the $\Delta_{0}$-Substitution Law, we have

$$
\llbracket \tilde{a}<v^{\prime} \rrbracket \wedge \llbracket v^{\prime} \leq \tilde{b} \rrbracket \wedge \llbracket u=v^{\prime} \rrbracket \leq \llbracket \tilde{a}<u \rrbracket \wedge \llbracket u \leq \tilde{b} \rrbracket
$$

for any $v^{\prime} \in \mathbf{R}^{(\mathcal{Q})}$, so that

$$
\llbracket u \in \tilde{I} \rrbracket \leq \llbracket \tilde{a}<u \rrbracket \wedge \llbracket u \leq \tilde{b} \rrbracket .
$$

From

$$
\begin{aligned}
\bigvee_{v^{\prime} \in \mathbf{R}^{(\mathcal{Q})}} \llbracket \tilde{a}<v^{\prime} \rrbracket \wedge \llbracket v^{\prime} \leq \tilde{b} \rrbracket \wedge \llbracket u=v^{\prime} \rrbracket & \geq \llbracket \tilde{a}<u \rrbracket \wedge \llbracket u \leq \tilde{b} \rrbracket \wedge \llbracket u=u \rrbracket \\
& =\llbracket \tilde{a}<u \rrbracket \wedge \llbracket u \leq \tilde{b} \rrbracket
\end{aligned}
$$


we have

$$
\llbracket u \in \tilde{I} \rrbracket \geq \llbracket \tilde{a}<u \rrbracket \wedge \llbracket u \leq \tilde{b} \rrbracket .
$$

Hence, we have

$$
\llbracket u \in \tilde{I} \rrbracket=\llbracket \tilde{a}<u \rrbracket \wedge \llbracket u \leq \tilde{b} \rrbracket
$$

for any $u \in \mathbf{R}^{(\mathcal{Q})}$. Let $A \eta \mathcal{Q}^{\prime \prime}$ be a self-adjoint operator. From Proposition 5.11, we have

$$
E^{A}(\lambda)=\bigwedge_{\lambda<x \in \mathbf{Q}} \tilde{A}(\check{x})=\llbracket \tilde{A} \leq \tilde{\lambda} \rrbracket
$$

for any $\lambda \in \mathbf{R}$. Thus, we have

$$
\begin{aligned}
E^{A}(I) & =E^{A}(b)-E^{A}(a)=E^{A}(b) \wedge E^{A}(a)^{\perp}=\llbracket \tilde{A} \leq \tilde{b} \wedge \neg(\tilde{A} \leq \tilde{a}) \rrbracket=\llbracket \tilde{a}<\tilde{A} \leq \tilde{b} \rrbracket \\
& =\llbracket \tilde{A} \in \tilde{I} \rrbracket .
\end{aligned}
$$

Thus, the assertion follows. QED

Let $\mathcal{Q}$ be a $\operatorname{logic}$ on $\mathcal{H}$. Any unit vector $\psi \in \mathcal{H}$ is called a (vector) state of $\mathcal{Q}$. We define the probability of any statement $\phi$ in $\mathcal{L}\left(\in, V^{(\mathcal{Q})}\right)$ in a state $\psi$ by

$$
\operatorname{Pr}\{\phi \| \psi\}=\|\llbracket \phi \rrbracket \psi\|^{2} .
$$

We say that statement $\phi$ in $\mathcal{L}\left(\in, V^{(\mathcal{Q})}\right)$ holds in state $\psi$ iff $\operatorname{Pr}\{\phi \| \psi\}=1$, and this condition is equivalent to $\psi \in \mathcal{R} \llbracket \phi \rrbracket$. In what follows, we shall show that this probabilistic interpretation of the statements in $\mathcal{L}\left(\in, V^{(\mathcal{Q})}\right)$ is consistent with the standard formulation of quantum mechanics.

In the standard formulation of (non-relativistic) quantum mechanics, every quantum system $\mathbf{S}$ corresponds to a Hilbert space $\mathcal{H}$. An observable of $\mathbf{S}$ is represented by a selfadjoint operator (densely defined) on $\mathcal{H}$, and a (vector) state of $\mathbf{S}$ is represented by a unit vector $\psi \in \mathcal{H}$.

For any observable $A$, let $E^{A}(\lambda)$ be the resolution of the identity belonging to $A$. A basic principle of quantum mechanics is formulated as follows [41, p. 200]. In the state $\psi$ mutually commuting observables $A_{1}, \ldots, A_{n}$ take values from the respective intervals $I_{1}, \ldots, I_{n}$ with the probability

$$
\left\|E^{A_{1}}\left(I_{1}\right) \cdots E^{A_{n}}\left(I_{n}\right) \psi\right\|^{2}
$$

Let $\tilde{A}_{1}, \ldots, \tilde{A}_{n}$ be the corresponding elements in $V^{(\mathcal{Q}(\mathcal{H}))}$, and $\tilde{I}_{1}, \ldots, \tilde{I}_{n}$ the corresponding intervals in $V^{(\mathcal{Q}(\mathcal{H}))}$. Then, from Theorem 6.2 we have

$$
\begin{aligned}
\operatorname{Pr}\left\{\tilde{A}_{1} \in \tilde{I}_{1} \wedge \cdots \wedge \tilde{A}_{n} \in \tilde{I}_{n} \| \psi\right\} & =\left\|\llbracket \tilde{A}_{1} \in \tilde{I}_{1} \wedge \cdots \wedge \tilde{A}_{n} \in \tilde{I}_{n} \rrbracket \psi\right\|^{2} \\
& =\left\|E^{A_{1}}\left(I_{1}\right) \cdots E^{A_{n}}\left(I_{n}\right) \psi\right\|^{2}
\end{aligned}
$$

Thus, we can restate the basic principle of quantum mechanics as follows. In the state $\psi$ mutually commuting observables $A_{1}, \ldots, A_{n}$ take values from the respective intervals $I_{1}, \ldots, I_{n}$ with the probability

$$
\operatorname{Pr}\left\{\tilde{A}_{1} \in \tilde{I}_{1} \wedge \cdots \wedge \tilde{A}_{n} \in \tilde{I}_{n} \| \psi\right\}
$$


Therefore, we have shown that there is a natural one-to-one correspondence between observables of a quantum system described by a Hilbert space $\mathcal{H}$ and real numbers in the universe $V^{(\mathcal{Q}(\mathcal{H}))}$ of quantum sets, so that observational propositions on the quantum system is naturally expressed as the valid statements on the real numbers in $V^{(\mathcal{Q}(\mathcal{H}))}$.

In the conventional interpretation of quantum mechanics [41], atomic observational propositions are restricted to those of the form $\tilde{A} \in \tilde{I}$ for an observable $A$ and an interval $\tilde{I}$ as above. However, quantum set theory is expected to extend the interpretation of quantum mechanics to a more general class of observational propositions. Here, we introduce one such extension of the interpretation.

For any two commuting observables $A$ and $B$ and any state $\psi$, we have a joint probability distribution $\mu_{\psi}^{A, B}$ of $A$ and $B$ in $\psi$, a probability measure on $\mathbf{R}^{2}$ satisfying

$$
\mu_{\psi}^{A, B}(I \times J)=\operatorname{Pr}\{\tilde{A} \in \tilde{I} \wedge \tilde{B} \in \tilde{J} \| \psi\}=\left\|E^{A}(I) E^{B}(J) \psi\right\|^{2}
$$

for any intervals $I$ and $J$. Then, it is natural to consider that $A$ and $B$ have the same value in state $\psi$ if and only if

$$
\operatorname{Pr}\{\tilde{A} \in \tilde{I} \wedge \tilde{B} \in \tilde{J} \| \psi\}=0
$$

for any $I, J$ such that $I \cap J=\emptyset$, and moreover this condition is equivalent to the following conditions:

(i) $\mu_{\psi}^{A, B}\left(\left\{(a, b) \in \mathbf{R}^{2} \mid a=b\right\}\right)=1$.

(ii) $\mu_{\psi}^{A, B}\left(\left\{(a, b) \in \mathbf{R}^{2} \mid a \neq b\right\}\right)=0$.

(iii) $\mu_{\psi}^{A, B}(I \times J)=\mu_{\psi}^{A, B}((I \cap J) \times \mathbf{R})=\mu_{\psi}^{A, B}(\mathbf{R} \times(I \cap J))$ for any intervals $I$ and $J$. Following the classical probability theory, we say in this case that observables $A$ and $B$ are perfectly correlated in state $\psi$. Thus, the notion of perfect correlation is straightforward for any pair of commuting observables. However, the problem of extending this notion to any pair of non-commuting observables has a non-trivial difficulty, since we have no universal definition of the joint probability distribution for noncommuting observables.

In the recent investigations [26, 27], we have obtained a satisfactory solution for the above problem. Here, we shall consider this problem in the light of quantum set theory. Since $\llbracket \tilde{A} \leq \tilde{r} \rrbracket=\llbracket \check{r} \in \tilde{A} \rrbracket$ for any $r \in \mathbf{Q}$, it is natural to say that $A$ and $B$ have the same value in state $\psi$ iff $\psi \in \mathcal{R} \llbracket(\forall r \in \check{Q}) r \in \tilde{A} \leftrightarrow r \in \tilde{B} \rrbracket$, or equivalently iff $\psi \in \mathcal{R} \llbracket \tilde{A}=\tilde{B} \rrbracket$.

Now, we shall show that the above condition is equivalent to the notion of perfect correlation formulated in [26, 27]. Let $A$ be an observable. For any (complex-valued) bounded Borel function $f$ on $\mathbf{R}$, we define the observable $f(A)$ by

$$
f(A)=\int_{\mathbf{R}} f(\lambda) d E^{A}(\lambda) .
$$

We shall denote by $B(\mathbf{R})$ the space of bounded Borel functions on $\mathbf{R}$. For any Borel set $\Delta$ in $\mathbf{R}$, we define $E^{A}(\Delta)$ by $E^{A}(\Delta)=\chi_{\Delta}(A)$, where $\chi_{\Delta}$ is a Borel function on $\mathbf{R}$ defined by $\chi_{\Delta}(x)=1$ iff $x \in \Delta$ and $\chi_{\Delta}(x)=0$ iff $x \notin \Delta$. For any pair of observables $A$ and $B$, the joint probability distribution of $A$ and $B$ in a state $\psi$ is a probability measure $\mu_{\psi}^{A, B}$ on $\mathbf{R}^{2}$ satisfying

$$
\mu_{\psi}^{A, B}(\Delta \times \Gamma)=\left\langle\psi,\left(E^{A}(\Delta) \wedge E^{B}(\Gamma)\right) \psi\right\rangle
$$


for any $\Delta, \Gamma \in \mathcal{B}(\mathbf{R})$. Gudder [8] showed that the joint probability distribution $\mu_{\psi}^{A, B}$ exists if and only if the relation $\left[E^{A}(\Delta), E^{B}(\Gamma)\right] \psi=0$ holds for every $\Delta, \Gamma \in \mathcal{B}(\mathbf{R})$.

Theorem 6.3 For any observables (self-adjoint operators) $A, B$ on $\mathcal{H}$ and any state (unit vector) $\psi \in \mathcal{H}$, the following conditions are all equivalent.

(i) $\psi \in \mathcal{R} \llbracket \tilde{A}=\tilde{B} \rrbracket$.

(ii) $E^{A}(r) \psi=E^{B}(r) \psi$ for any $r \in \mathbf{Q}$.

(iii) $f(A) \psi=f(B) \psi$ for all $f \in B(\mathbf{R})$.

(iv) $\left\langle E^{A}(\Delta) \psi, E^{B}(\Gamma) \psi\right\rangle=0$ for any $\Delta, \Gamma \in \mathcal{B}(\mathbf{R})$ with $\Delta \cap \Gamma=\emptyset$.

(v) There is the joint probability distribution $\mu_{\psi}^{A, B}$ of $A$ and $B$ in $\psi$ satisfying

$$
\mu_{\psi}^{A, B}\left(\left\{(a, b) \in \mathbf{R}^{2} \mid a=b\right\}\right)=1
$$

Proof. The equivalence (i) $\Leftrightarrow$ (ii) follows from Theorem 5.6. Suppose that (ii) holds. Let $\lambda \in \mathbf{R}$. If $r_{1}, r_{2}, \ldots$ be a decreasing sequence of rational numbers convergent to $\lambda$, then $E^{A}\left(r_{n}\right) \psi$ and $E^{B}\left(r_{n}\right) \psi$ are convergent to $E^{A}(\lambda) \psi$ and $E^{B}(\lambda) \psi$, respectively, so that $E^{A}(\lambda) \psi=E^{B}(\lambda) \psi$ for all $\lambda \in \mathbf{R}$. Thus, we have

$$
\langle\xi, f(A) \psi\rangle=\int_{\mathbf{R}} f(\lambda) d\left\langle\xi, E^{A}(\lambda) \psi\right\rangle=\int_{\mathbf{R}} f(\lambda) d\left\langle\xi, E^{B}(\lambda) \psi\right\rangle=\langle\xi, f(B) \psi\rangle
$$

for all $\xi \in \mathcal{H}$, and hence we have $f(A) \psi=f(B) \psi$ for all $f \in B(\mathbf{R})$. Thus, the implication (ii) $\Rightarrow$ (iii) holds. Since condition (ii) is a special case of condition (iii) where $f=\chi_{(-\infty, r]}$, the implication (iii) $\Rightarrow$ (ii) is trivial, so that the equivalence (ii) $\Leftrightarrow$ (iii) follows. The equivalence of assertions (iii), (iv), and (v) have been already proved in Ref. [27, the proof is completed. $Q E D$

Condition (iv) above is adopted as the defining condition for $A$ and $B$ to be perfectly correlated in $\psi$ because of the simplicity and generality of the formulation. Condition (v) justifies our nomenclature calling $A$ and $B$ "perfectly correlated." By condition (i), quantum logic justifies the assertion that "perfectly correlated" observables actually have the same value in the given state. For further properties and applications of the notion of perfect correlation, we refer the reader to Ref. [27].

\section{Concluding Remarks}

In classical logic, the implication connective $\rightarrow$ is defined by negation and disjunction as $P \rightarrow Q=(\neg P) \vee Q$. In quantum logic several counterparts have been proposed. Hardegree [10] proposed the following requirements for the implication connective.

(E) $P \rightarrow Q=1$ if and only if $P \leq Q$.

$(\mathrm{MP}) P \wedge(P \rightarrow Q) \leq Q$.

(MT) $Q^{\perp} \wedge(P \rightarrow Q) \leq P^{\perp}$.

(LB) If $P ! Q$, then $P \rightarrow Q=P^{\perp} \vee Q$.

Then, the work of Kotas [13] can be applied to the problem as to what complementedlattice-polynomial definitions of $P \rightarrow Q$ satisfy the above conditions; there are exactly three possibilities:

(i) $P \rightarrow_{1} Q=P^{\perp} \vee(P \wedge Q)$. 
(ii) $P \rightarrow_{2} Q=(P \vee Q)^{\perp} \vee Q$.

(iii) $P \rightarrow_{3} Q=(P \wedge Q) \vee\left(P^{\perp} \wedge Q\right) \vee\left(P^{\perp} \wedge Q^{\perp}\right)$.

However, so far we have no general agreement on the choice from the above, although the majority view favors definition (i), the so-called Sasaki arrow [40].

In quantum set theory, the truth values of atomic formulas, $\llbracket u \in v \rrbracket$ and $\llbracket u=v \rrbracket$, depend crucially on the definition of the implication connective. Takeuti 35] chose the Sasaki arrow for this and the present work has followed Takeuti's choice.

Here, another approach should be also mentioned. Titani and Kozawa [39] developed "quantum set theory" based on the implication connective defined by $P \rightarrow Q=1$ if $P \leq Q$ and $P \rightarrow Q=0$ otherwise. Then, their implication connective satisfies (E), (MP), and (MT), but does not satisfy (LB). They successfully showed that there is a one-to-one correspondence between quantum reals and observables. However, their truth values of relations between quantum reals is not consistent with the standard interpretation of quantum mechanics. In fact, for any observable $A$ and any real number $a \in \mathbf{R}$, their truth value of the relation $\tilde{A} \leq \tilde{a}$ satisfies $\llbracket \tilde{A} \leq \tilde{a} \rrbracket=1$ if $A \leq a 1$ and $\llbracket \tilde{A} \leq \tilde{a} \rrbracket=0$ otherwise. Moreover, their equality between two quantum reals takes only two values so that $\llbracket \tilde{A}=\tilde{B} \rrbracket=1$ if $A=B$ and $\llbracket \tilde{A}=\tilde{B} \rrbracket=0$ otherwise. Thus, the Titani-Kozawa approach does not lead to consistency results similar to Theorem 6.2 or Theorem 6.3.

Although the choice of the implication connective affects the truth value assignment of any statements in the language of set theory, it is natural to expect that not only the implication connective (i) but also the implication connectives (ii) and (iii) will lead to consistency results similar to the above mentioned results. Thus, it is an interesting open problem to figure out the characteristic differences in quantum set theories with different choices of the implication connective from (i), (ii) and (iii) above. From the investigations in this line, it is expected to find a reasonable affirmative answer to the question "Is a quantum logic a logic?" [7].

\section{Acknowledgments}

This work was supported by the SCOPE project of MIC of Japan and the Grant-in-Aid for Scientific Research of the JSPS.

\section{References}

[1] J. L. Bell, Boolean-valued models and independence proofs in set theory, 2nd ed., Oxford University Press, Oxford, 1985.

[2] G. Birkhoff and J. von Neumann, The logic of quantum mechanics, Ann. Math. 37 (1936), 823-845.

[3] O. Bratteli and D. W. Robinson, Operator algebras and quantum statistical mechanics I, Springer, New York, 1979.

[4] P. J. Cohen, The independence of the continuum hypothesis I, Proc. Nat. Acad. Sci. U.S.A. 50 (1963), 1143-1148.

[5] _ Set theory and the continuum hypothesis, Benjamin, New York, 1966. 
[6] K. Eda, On a Boolean power of a torsion free abelian group, J. Algebra 82 (1983), 84-93.

[7] G. J. Greechie and S. P. Gudder, Is a quantum logic a logic?, Helv. Phys. Acta 41 (1971), 238-240.

[8] S. Gudder, Joint distributions of observables, J. Math. Mech. 18 (1968), 325-335.

[9] G. M. Hardegree, The conditional in abstract and concrete quantum logic, The logicoalgebraic approach to quantum mechanics, Volume II: Contemporary consolidation (C. A. Hooker, ed.), D. Reidel, Dordrecht, 1979, pp. 49-108.

[10] _ Material implication in orthomodular (and Boolean) lattices, Notre Dame J. Formal Logic 22 (1981), 163-182.

[11] T. Jech, Abstract theory of abelian operator algebras: an application of forcing, Trans. Amer. Math. Soc. 289 (1985), 133-162.

[12] G. Kalmbach, Orthomodular lattices, Academic, London, 1983.

[13] J. Kotas, An axiom system for the modular logic, Studia Logica 21 (1967), 17-38.

[14] A. G. Kusraev and S. S. Kutateladze, Nonstandard methods of analysis, Springer, Berlin, 1994.

[15] _ Boolean valued analysis, Springer, Berlin, 1999.

[16] H. Nishimura, Boolean valued Lie algebras, J. Symbolic Logic 56 (1991), 731-741.

[17] M. Ozawa, Boolean valued analysis and type I $A W^{*}$-algebras, Proc. Japan Acad. 59 A (1983), 368-371.

[18] _ Boolean valued interpretation of Hilbert space theory, J. Math. Soc. Japan 35 (1983), 609-627.

[19] _ A classification of type I AW*-algebras and Boolean valued analysis, J. Math. Soc. Japan 36 (1984), 589-608.

[20] - Nonuniqueness of the cardinality attached to homogeneous $A W^{*}$-algebras, Proc. Amer. Math. Soc. 93 (1985), 681-684.

[21] _ A transfer principle from von Neumann algebras to $A W^{*}$-algebras, J. London Math. Soc. (2) 32 (1985), 141-148.

[22] _ Boolean valued analysis approach to the trace problem of $A W^{*}$-algebras, J. London Math. Soc. (2) 33 (1986), 347-354.

[23] _ Boolean valued interpretation of Banach space theory and module structures of von Neumann algebras, Nagoya Math. J. 117 (1990), 1-36.

[24] 263-297. 
[25] _ Scott incomplete Boolean ultrapowers of the real line, J. Symbolic Logic 60 (1995), 160-171.

[26] _ Perfect correlations between noncommuting observables, Phys. Lett. A 335 (2005), 11-19.

[27] — Quantum perfect correlations, Ann. Phys. (N.Y.) 321 (2006), 744-769.

[28] D. Scott, Boolean models and nonstandard analysis, Applications of Model Theory to Algebra, Analysis, and Probability (W. A. J. Luxemburg, ed.), Holt, Reinehart and Winston, New York, 1969, pp. 87-92.

[29] D. Scott and R. Solovay, Boolean-valued models for set theory, unpublished manuscript for Proc. AMS Summer Institute on Set Theory, Los Angeles: Univ. Cal., 1967.

[30] K. Smith, Commutative regular rings and Boolean-valued fields, J. Symbolic Logic 49 (1984), 281-297.

[31] M. Takesaki, Theory of operator algebras I, Springer, New York, 1979.

[32] G. Takeuti, Two applications of logic to mathematics, Princeton University Press, Princeton, 1978.

[33] _ Boolean valued analysis, Applications of Sheaves (Fourman, Mulvey, and Scott, eds.), Lecture Notes in Math. 753, Springer, Berlin, 1979, pp. 714-731.

[34] _ A transfer principle in harmonic analysis, J. Symbolic Logic 44 (1979), $417-440$.

[35] _ Quantum set theory, Current Issues in Quantum Logic (London) (Beltrametti, ed.), Plenum, 1981, pp. 303-322.

[36] — $C^{*}$-Algebras and Boolean valued analysis, Japan. J. Math. 9 (1983), 207-245.

[37] _ Von Neumann algebras and Boolean valued analysis, J. Math. Soc. Japan 35 (1983), 1-21.

[38] _ Boolean simple groups and Boolean simple rings, J. Symbolic Logic 53 (1988), 160-173.

[39] S. Titani and H. Kozawa, Quantum set theory, Int. J. Theor. Phys. 42 (2003), 25752602.

[40] A. Urquhart, Review, J. Symbolic Logic 48 (1983), 206-208.

[41] J. von Neumann, Mathematical foundations of quantum mechanics, Princeton UP, Princeton, NJ, 1955, [English translation of Mathematische Grundlagen der Quantenmechanik (Springer, Berlin, 1932)]. 